\title{
A FUNCTION SPACE MODEL APPROACH TO THE RATIONAL EVALUATION SUBGROUPS
}

\author{
YOSHIHIRO HIRATO, KATSUHIKO KURIBAYASHI AND NOBUYUKI ODA
}

\begin{abstract}
Let $f: U \rightarrow X$ be a map from a connected nilpotent space $U$ to a connected rational space $X$. The evaluation subgroup $G_{*}(U, X ; f)$, which is a generalization of the Gottlieb group of $X$, is investigated. The key device for the study is an explicit Sullivan model for the connected component containing $f$ of the function space of maps from $U$ to $X$, which is derived from the general theory of such a model due to Brown and Szczarba [5]. In particular, we show that non Gottlieb elements are detected by analyzing a Sullivan model for the map $f$ and by looking at non-triviality of higher order Whitehead products in the homotopy group of $X$. The Gottlieb triviality of a fibration in the sense of Lupton and Smith [27] is also discussed from the function space model point of view. Moreover, we proceed to consideration of the evaluation subgroup of the fundamental group of a nilpotent space. In consequence, the first Gottlieb group of the total space of each $S^{1}$-bundle over the $n$-dimensional torus is determined explicitly in the non-rational case.
\end{abstract}

\section{INTRODUCTION}

Let $U$ and $X$ be connected based spaces and $f: U \rightarrow X$ a based map. We denote by $\mathcal{F}(U, X ; f)$ the connected component in the function space of free maps from $U$ to $X$ that contains $f$. Let $e v: \mathcal{F}(U, X ; f) \rightarrow X$ be the evaluation map which sends a map $g: U \rightarrow X$ to $g\left(u_{0}\right)$, where $u_{0}$ is the base point of $U$. The $n$th evaluation subgroup for the triple $(U, X ; f)$, denoted $G_{n}(U, X ; f)$, is the subgroup of the homotopy group $\pi_{n}(X)$ defined by

$$
G_{n}(U, X ; f)=e v_{*}\left(\pi_{n}(\mathcal{F}(U, X ; f), f)\right) .
$$

In the special case where $U=X$ and $f=i d$ the identity map on $X$, the $n$th evaluation subgroup is referred to as the $n$th Gottlieb group of $X$ and written $G_{n}(X)$. In what follows, we shall write $G_{*}(U, X ; f)$ for $\oplus_{n \geq 0} G_{n}(U, X ; f)$.

The evaluation subgroups were essentially introduced by Gottlieb [12][14] and were investigated extensively by Woo and Kim [38] [39] and by Woo and Lee [23] [40] [41] [42]. The lack of functoriality in Gottlieb groups makes the study of the subject more difficult. In such a situation, the $G$-sequence introduced in [41] is one of relevant tools for studying the groups $G_{*}(X)$ and $G_{*}(U, X ; f)$.

2000 Mathematics Subject Classification: 55P62, 55Q70.

Key words and phrases. Evaluation subgroup, Gottlieb group, Whitehead product, Sullivan model.

Department of Mathematical Sciences, Faculty of Science, Shinshu University, Matsumoto, Nagano 390-8621, Japan e-mail:hirato@math.shinshu-u.ac.jp

Department of Mathematical Sciences, Faculty of Science, Shinshu University, Matsumoto, Nagano 390-8621, Japan e-mail:kuri@math.shinshu-u.ac.jp

Department of Applied Mathematics, Faculty of Science, Fukuoka University, Fukuoka 8140180, Japan e-mail:odanobu@cis.fukuoka-u.ac.jp 
As for rational Gottlieb groups, Félix and Halperin have proved that, for any simply-connected space $X$ with finite rational Lusternik-Schnirelmann category $m$, the graded Gottlieb group $G_{*}(X) \otimes \mathbb{Q}$ is concentrated in odd degrees and has dimension at most $m([8$, Theorem III $])$. We stress that the consideration of Gottlieb groups appears in their investigation of rational category. Moreover, from the lecture notes [30] due to Oprea, we can know relationship between Gottlieb groups and transformation groups as well as fixed point theory. In [35], Smith has studied the rational evaluation subgroups by relying on the approach to the study of function spaces due to Federer [7]. Interesting examples of vanishing and non-vanishing evaluation subgroups are given in [35, §5]. Recently, Lupton and Smith [25][26] have considered the exactness of the $G$-sequence by representing the evaluation subgroups in terms of derivations in Sullivan models and in Quillen models. Especially, in [25, Example 4.1], the non-exactness of a certain $G$-sequence is captured by calculation of derivations.

The objective of this paper is to investigate the evaluation subgroup $G_{*}\left(U, X_{\mathbb{Q}} ; f\right)$, where $U$ is a nilpotent space and $X_{\mathbb{Q}}$ is the localization of a nilpotent space $X$. We try to consider the rational evaluation subgroup without drawing on the derivation argument. In fact, the key device for the study is an explicit algebraic model for the function space $\mathcal{F}\left(U, X_{\mathbb{Q}} ; f\right)$, which we construct in this paper by invoking the general theory of such a model due to Brown and Szczarba [5]; see Section 3.

We here explain our main results briefly. Theorems 1.1 and 1.2 describe sufficient conditions for rational evaluation subgroups to be proper. Theorem 1.6 presents a tractable condition for a fibration to be Gottlieb trivial in the sense of Lupton and Smith [27]. Theorem 1.7 gives a non-trivial upper bound for the dimension of the localization of some subquotient of the first evaluation subgroup. By Theorem 1.9, one can determine the first Gottlieb group of the total space of each $S^{1}$-bundle over the $n$-dimensional torus in non-rational case with knowledge of the classifying map of the bundle.

Unless otherwise explicitly stated, it is assumed that a space is well-based and has the homotopy type of a CW complex with rational homology of finite type. We further suppose that a map is based. We shall say that a space is rational if the space has the homotopy type of the spatial realization of a Sullivan algebra; see Section 2. Observe that the homotopy group $\pi_{n}(X)$ of a rational space $X$ is a vector space over $\mathbb{Q}$ for $n>1$ and that so is the fundamental group $\pi_{1}(X)$ if the group is abelian. These facts follow from the Sullivan-de Rham equivalence; see for example [2, Theorems 10.1 and 12.2].

In the rest of this section, we state the results more precisely.

Suppose that $X$ is a connected rational space. Then the function space $\mathcal{F}(U, X ; f)$ is also a rational space; see (2.2) and (2.3) in Section 2. The definition of the evaluation subgroup enables us to obtain a commutative diagram

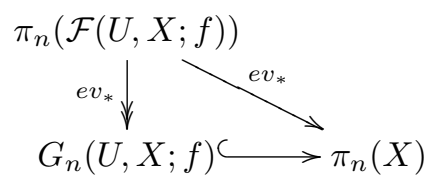

in the category of groups for $n \geq 1$. This is regarded as a diagram in the category of vector spaces for $n>1$. Let $H$ be a group and let $\left(\Gamma_{1} / \Gamma_{2}\right) H$ denote the quotient group of $H$ by the commutator subgroup. Put $G^{\sharp}=\operatorname{Hom}_{\mathbb{Z}}(G, \mathbb{Q})$ for an abelian 
group $G$. Then we have a commutative diagram

$$
\left(\left(\Gamma_{1} / \Gamma_{2}\right) \pi_{n}(\mathcal{F}(U, X ; f))\right)_{G_{n}(U, X ; f)^{\sharp} \ll}^{\sharp} \pi_{n}(X)^{\sharp}
$$

in the category of vector spaces for $n>1$ and for $n=1$ if $\pi_{1}(X)$ is abelian. Recall that, for any connected nilpotent space $Y$ with a minimal model $\wedge Z$, there exists a natural isomorphism $Z^{n} \cong \pi_{n}(Y)^{\sharp}$ for $n>1$ and for $n=1$ if $\pi_{1}(Y)$ is an abelian group; see [2].

Let $\wedge V$ and $\wedge W$ be minimal models for $X$ and $\mathcal{F}(U, X ; f)$, respectively. We denote by $Q(\widetilde{e v}): V \rightarrow W$ the linear part of the Sullivan representative $\widetilde{e v}: \wedge V \rightarrow$ $\wedge W$ for the evaluation map. Observe that the vector space $\left(\left(\Gamma_{1} / \Gamma_{2}\right) \pi_{n}(\mathcal{F}(U, X ; f))\right)^{\#}$ is a subspace of $W^{n}$; see Section 8 for details. Suppose that $\pi_{1}(X)$ is an abelian group. Then we have an isomorphism $G_{*}(U, X ; f)^{\sharp} \cong V / \operatorname{Ker} Q(\widetilde{e v})$ of vector spaces. This fact implies, for example, that $G_{*}(U, X ; f)$ is a proper subgroup of $\pi_{*}(X)$ if and only if $\operatorname{Ker} Q(\widetilde{e v})$ is nontrivial.

In [5], Brown and Szczarba have presented an explicit form of Lannes' division functor in the category of commutative differential graded algebras; see also [3]. By using the functor, they have constructed an algebraic model for a connected component of a function space. Unfortunately, the model is very complicated and not minimal in general. However the linear part $\delta_{0}$ of the differential of the model for $\mathcal{F}(U, X ; f)$, which is needed to construct the minimal model, is comparatively

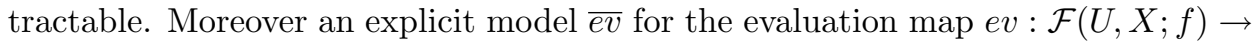
$X$ is derived from the consideration in [21, Section 5].

In some cases, we can find a nonzero element in $\operatorname{Im} \overline{e v} \cap \operatorname{Im} \delta_{0}$ with knowledge of the terms having the least wordlength in $d(v)$ for an appropriate element $v \in V$. It turns out then that $\operatorname{Ker} Q(\widetilde{e v}) \neq 0$. The dual element in $V^{\sharp} \cong \pi_{*}(X)$ to such an element $v$ is said to be detective; see Section 4 for the precise definition. With this terminology, one of our main theorems is stated as follows.

Theorem 1.1. Let $f: U \rightarrow X$ be a map from a connected nilpotent space $U$ to a connected rational space $X$ whose fundamental group is abelian. Suppose that $\operatorname{dim} \oplus_{q \geq 0} H^{q}(U ; \mathbb{Q})<\infty$ or $\operatorname{dim} \oplus_{i \geq 2} \pi_{i}(X)<\infty$ and that there exists a detective element $x$ in $\pi_{*}(X)$ with respect to the triple $(U, X ; f)$. Then the evaluation subgroup $G_{k}(U, X ; f)$ is a proper subgroup of $\pi_{k}(X)$ for some $1 \leq k \leq \operatorname{deg} x$.

While the notion of the detective element is somewhat technical, it does work well when exhibiting the properness of a given evaluation subgroup; see Example 4.6 .

We can also detect geometrically an element which is not in the evaluation subgroup. Before describing the result, we recall briefly the higher order Whitehead product set defined by Porter in [32]. Let $\iota_{m}$ denote the generator of $H_{m}\left(S^{m}\right)$ which is the image of the identity map by the Hurewicz map. Let $T$ be the fat wedge of $s$ spheres $S^{n_{i}}, 1 \leq i \leq s$; that is, the subspace of the product $S^{n_{1}} \times \cdots \times S^{n_{s}}$ consisting of all $s$-tuples with at least one coordinate at the base point. Let $\mu$ be the generator of $H_{N}\left(\times_{i=1}^{s} S^{n_{i}} ; \mathbb{Z}\right)$, corresponding to $\iota_{n_{1}} \otimes \cdots \otimes \iota_{n_{s}} \in H_{*}\left(S^{n_{1}}\right) \otimes \cdots \otimes H_{*}\left(S^{n_{s}}\right)$ via the Künneth isomorphism, where $N=\sum n_{i}$. Since the CW pair $\left(\times_{i=1}^{s} S^{n_{i}}, T\right)$ is 
$(N-1)$-connected, we have a sequence

$$
H_{N}\left(\times_{i=1}^{s} S^{n_{i}}\right) \stackrel{j_{*}}{\longrightarrow} H_{N}\left(\times_{i=1}^{s} S^{n_{i}}, T\right) \stackrel{h}{\cong} \pi_{N}\left(\times_{i=1}^{s} S^{n_{i}}, T\right) \stackrel{\partial}{\longrightarrow} \pi_{N-1}(T)
$$

and an element $w=\partial h^{-1} j_{*}(\mu)$, where $h$ is the Hurewicz map and $\partial$ is the boundary map. In what follows, we do not distinguish between a map and the homotopy class which it represents. Choose elements $x_{i} \in \pi_{n_{i}}(X)$ for $1 \leq i \leq s$. These elements define the map $g: \vee_{i=1}^{s} S^{n_{i}} \rightarrow X$ whose restriction to each $S^{n_{i}}$ is the map $x_{i}$. Then the $s$ th order Whitehead product set $\left[x_{1}, \ldots, x_{s}\right] \subset \pi_{N-1}(X)$ (possibly empty) is defined by

$$
\left[x_{1}, \ldots, x_{s}\right]=\left\{f_{*}(w) \mid f: T \rightarrow X \text { an extension of } g\right\} .
$$

We shall say that the set $\left[x_{1}, \ldots, x_{s}\right]$ vanishes if it contains only zero.

As a consequence of a geometric property of higher-order Whitehead products in rational spaces, studied in [1], we obtain the following test for non-Gottlieb elements.

Theorem 1.2. Let $U$ be a connected space and $X$ a simply-connected rational space. Let $f: U \rightarrow X$ be a map for which the induced map $f_{*}: \pi_{*}(U) \rightarrow \pi_{*}(X)$ is an epimorphism. Assume that all Whitehead products of order less than $r$ vanish in $\pi_{*}(U)$. If there exist elements $x_{1}, \ldots, x_{r}$ in $\pi_{*}(X)$ whose rth order Whitehead product $\left[x_{1}, \ldots, x_{r}\right]$ contains a nonzero element, then $x_{k} \notin G_{*}(U, X ; f)$ for any $k \leq r$.

Remark 1.3. The result [1, Corollary 6.5] asserts that, if all Whitehead products of order $<r$ vanish in $\pi_{*}(X)$ for a simply-connected rational space $X$, then any $r$ th order Whitehead product sets in $\pi_{*}(X)$ is non-empty and consists of a single element. Therefore the Whitehead product $\left[x_{1}, \ldots, x_{r}\right]$ in Theorem 1.2 contains only one element.

Suppose that $x_{1}$ is a Gottlieb element in $\pi_{*}(X)$ for a connected space $X$ which is not necessarily rational. The ordinary Whitehead product $\left[x_{1}, x_{2}\right]$ is zero for any $x_{2} \in \pi_{*}(X)$ by [14, Proposition 2.3]. Thus Theorem 1.2 is regarded as a generalization of this fact in the context of rational homotopy theory.

It is worthwhile to deal with relationship between detective elements and higher order Whitehead product sets. With the aid of results in [1], we shall show that a nonzero element in a higher order Whitehead product set is detective; see Theorem 6.1 .

As described below, the sufficient conditions in Theorems 1.1 and 1.2 give criterions for a map not to be cyclic.

For maps $f: U \rightarrow X$ and $g: V \rightarrow X$, we write $g \perp f$ if the map $g \vee f: V \vee U \rightarrow X$ is extendable to $V \times U$. A map $f: U \rightarrow X$ is called a cyclic map if $i d_{X} \perp f$. For example, when a topological group $G$ acts on a space X with base point, the orbit map $G \rightarrow X$ at the base point is a cyclic map. As is discussed in the last paragraph on page 730 of [14], we see that $G_{n}(U, X ; f)=\left\{[g] \in \pi_{n}(X) \mid g \perp f\right\}$. It is readily seen that $\pi_{*}(X)=G_{*}(U, X ; f)$ if $f$ is a cyclic map. Observe that if $f$ is a cyclic map, then so is $e_{X} \circ f$, where $e_{X}: X \rightarrow X_{\mathbb{Q}}$ is the localization map. Thus we have the following corollary.

Corollary 1.4. Let $f: U \rightarrow X$ be a map between a connected nilpotent spaces and $e_{X}: X \rightarrow X_{\mathbb{Q}}$ the localization map. If the triple $\left(U, X_{\mathbb{Q}} ; e_{X} \circ f\right)$ satisfies the conditions in Theorem 1.1 or 1.2, then $f$ is not a cyclic map.

We fix some notations and terminology in order to describe further our results. 
Let $f: X \rightarrow Y$ be a map between nilpotent spaces. Let $\varphi:(\wedge V, d) \rightarrow A_{P L}(Y)$ be a minimal model for $Y$, where $A_{P L}(Y)$ denotes the differential graded algebra of rational polynomial forms on $Y$. A quasi-isomorphism $m:(\wedge V \otimes \wedge W, \widehat{d}) \rightarrow A_{P L}(X)$ is called a Sullivan model for $f$ if $\left.\widehat{d}\right|_{\wedge V}=d,\left.m\right|_{\wedge V}=A_{P L}(f) \varphi$ and there exists a well-ordered homogeneous basis $\left\{x_{\alpha}\right\}_{\alpha \in \mathcal{I}}$ of $W$ such that $\widehat{d}\left(1 \otimes x_{\alpha}\right) \in \wedge V \otimes \wedge\left(W_{\alpha}\right)$. Here $\wedge\left(W_{\alpha}\right)$ denotes the subalgebra generated by the $x_{\beta}$ with $\beta<\alpha$. We further assume, unless otherwise specified, that the model is minimal in the sense that $\operatorname{deg} x_{\beta}<\operatorname{deg} x_{\alpha}$ implies $\beta<\alpha$; see [16, 1.1 Definition] and [16, Theorems 6.1 and 6.2] for the existence and the uniqueness of a minimal Sullivan model for a map $f$. The inclusion $j:(\wedge V, d)>(\wedge V \otimes \wedge W, \widehat{d})$ is also referred to as a Sullivan model for $f$. Observe that the DGA $(\wedge V \otimes \wedge W, \widehat{d})$ is a Sullivan algebra; see $[9$, Proposition 15.5]. For a Sullivan algebra $A=(\wedge V, d)$, let $d_{0}$ denote the linear part of the differential $d$ and put

$$
\pi^{n}(A)=H^{n}\left(V, d_{0}\right)
$$

We define the $\psi$-homotopy space of $X$, denoted $\pi_{\psi}^{*}(X)$, to be the vector space $\pi^{*}(A)$ for which $A$ is a Sullivan model for $X$; see [16, Chapter 8]. Observe that $\pi_{\psi}^{*}$ gives rise to a functor from the category of connected spaces with Sullivan models to that of graded vector spaces over $\mathbb{Q}$. Moreover there exists a natural isomorphism $\pi_{\psi}^{*}(X) \cong \pi_{*}(X)^{\sharp}$ for $*>1$ and for $* \geq 1$ if $\pi_{1}(X)$ is abelian; see [2], [16]. For a free algebra $\wedge V$, let $\wedge^{\geq l} V$ denote the ideal generated by elements with word length greater than or equal to $l$.

We describe an important result concerning a decomposition of an evaluation subgroup. In [24], Woo and Lee show that, for any based spaces $F$ and $Y$,

$$
G_{*}(F, F \times Y ; i) \cong G_{*}(F) \oplus \pi_{*}(Y),
$$

where $i: F \rightarrow F \times Y$ denotes the inclusion into the first factor. This has motivated us to consider its generalization from the rational homotopy theory point of view.

We here introduce a class of maps.

Definition 1.5. A map $p: X \rightarrow Y$ is separable if there exists a Sullivan model $(\wedge V, d) \rightarrow(\wedge V \otimes \wedge W, \widehat{d})$ for $p$ such that

$$
\widehat{d}(w) \in \wedge^{\geq 2} V \otimes \wedge W+\mathbb{Q} \otimes \wedge^{\geq 2} W
$$

for any $w \in W$. A fibration $p: X \rightarrow Y$ is said to be separable if the projection $p$ is separable.

We establish the following theorem.

Theorem 1.6. Let $F \stackrel{i}{\rightarrow} X \stackrel{p}{\rightarrow} Y$ be a separable fibration of connected rational spaces with $\operatorname{dim} \oplus_{q \geq 0} H^{q}(F ; \mathbb{Q})<\infty$ or $\operatorname{dim} \oplus_{i \geq 2} \pi_{i}(X)<\infty$. Suppose that $F$ is simply-connected and $\pi_{1}(Y)$ acts on $H^{i}(F ; \mathbb{Q})$ nilpotently for any $i$. Then the sequence

$$
0 \rightarrow G_{n}(F) \stackrel{i_{\sharp}}{\rightarrow} G_{n}(F, X ; i) \stackrel{p_{\sharp}}{\rightarrow} \pi_{n}(Y) \rightarrow 0
$$

is exact for $n>1$.

Very recently, Lupton and Smith [27] have proved a similar result to Theorem 1.6. Let $F \stackrel{i}{\rightarrow} X \stackrel{p}{\rightarrow} Y$ be a fibration of simply-connected $\mathrm{CW}$ complexes. In the 
remarkable result [27, Theorem 5.3], a sufficient condition for the sequence

$$
0 \rightarrow G_{*}(F) \otimes \mathbb{Q} \stackrel{i_{\sharp} \otimes 1}{\rightarrow} G_{*}(F, X ; i) \otimes \mathbb{Q} \stackrel{p_{\sharp} \otimes 1}{\rightarrow} \pi_{*}(Y) \otimes \mathbb{Q} \rightarrow 0
$$

to be exact is described in terms of the classifying map of the fibration in the sense of Stasheff [37]. It is important to mention that Theorem 1.6 follows from [27, Theorems 4.2 and 5.3] provided the given fibration is the localization of a fibration $F \rightarrow X \rightarrow Y$ of simply-connected CW complexes of finite type with fibre $F$ finite. The fibration which yields the short exact sequence (1.2) is said to be Gottlieb trivial [27]. Theorem 1.6 asserts that the Gottlieb triviality of a fibration follows from the separability.

We turn our attention to the first evaluation subgroup of $\pi_{1}(X)$ for a nilpotent space $X$. When considering the subgroup, a detective element can be found with the knowledge of the minimal model for $X$, in particular, of the quadratic part of the differential if $\pi_{1}(X)$ is not abelian. This fact enables us to deduce Theorem 1.7 below.

Let $G$ be a nilpotent group with the lower central series

$$
G=\Gamma_{1} G \supset \Gamma_{2} G \supset \cdots \supset \Gamma_{j} G \supset \cdots,
$$

where, for $j \geq 2, \Gamma_{j} G=\left[G, \Gamma_{j-1} G\right]$ stands for the subgroup of $G$ generated by the commutators $\left\{x y x^{-1} y^{-1} \mid x \in G, y \in \Gamma_{j-1} G\right\}$. The nilpotency class of $G$, denoted $\operatorname{nil}(G)$, is defined to be the largest integer $c$ such that $\Gamma_{c} G \neq\{1\}$. We write $\left(\Gamma_{q} / \Gamma_{q+1}\right) G$ for the subquotient $\Gamma_{q} G / \Gamma_{q+1} G$.

Theorem 1.7. Let $f: U \rightarrow X$ be a map between a connected nilpotent spaces. Suppose that (i) $\pi_{\psi}^{1}(f): \pi_{\psi}^{1}(X) \rightarrow \pi_{\psi}^{1}(U)$ is a monomorphism and that (ii) $U$ is a finite $C W$ complex or $X$ is a rational space with $\operatorname{dim} \oplus_{i \geq 2} \pi_{i}(X)<\infty$.

(1) If $\left(\Gamma_{k} / \Gamma_{k+1}\right) \pi_{1}(X)^{\sharp} \neq 0$, then for any $i<k$,

$\operatorname{dim}\left(\Gamma_{i} G_{1}(U, X ; f) / \Gamma_{i+1} \pi_{1}(X) \cap \Gamma_{i} G_{1}(U, X ; f)\right) \otimes \mathbb{Q} \leq \operatorname{dim}\left(\Gamma_{i} / \Gamma_{i+1}\right) \pi_{1}(X) \otimes \mathbb{Q}-1$.

(2) If $\left(\left[\pi_{1}(X), \pi_{1}(X)\right] / \Gamma_{3} \pi_{1}(X)\right)^{\sharp} \neq 0$, then

$$
\operatorname{dim}\left(G_{1}(U, X ; f) /\left[\pi_{1}(X), \pi_{1}(X)\right] \cap G_{1}(U, X ; f)\right) \otimes \mathbb{Q} \leq \operatorname{dim} H_{1}(X ; \mathbb{Q})-2 .
$$

We see that the subgroup $\Gamma_{i} G_{1}(U, X ; f) / \Gamma_{i+1} \pi_{1}(X) \cap \Gamma_{i} G_{1}(U, X ; f)$ of the quotient group $\left(\Gamma_{i} / \Gamma_{i+1}\right) \pi_{1}(X)$ is proper for any $i \geq 1$ under the assumption in Theorem 1.7.

Corollary 1.8. If $G_{1}(U, X ; f)$ is abelian and $\left(\left[\pi_{1}(X), \pi_{1}(X)\right] / \Gamma_{3} \pi(X)\right)^{\sharp} \neq 0$, then $\operatorname{dim} G_{1}(U, X ; f) \otimes \mathbb{Q} \leq \operatorname{dim}\left(\left[\pi_{1}(X), \pi_{1}(X)\right] \cap G_{1}(U, X ; f)\right) \otimes \mathbb{Q}+\operatorname{dim} H_{1}(X ; \mathbb{Q})-2$.

If $g: S^{1} \rightarrow X$ is any map such that $[g] \in G_{1}(U, X ; f)$, then $g \perp f$. Hence, the result [29, Proposition 3.4 (1)] applies to an extension $\mu: S^{1} \times U \rightarrow X$ of $g \vee f: S^{1} \vee U \rightarrow X$. It follows that $[g] \cdot f_{*}(\alpha)=g_{*}\left(\left[i d_{S^{1}}\right]\right) \cdot f_{*}(\alpha)=f_{*}(\alpha) \cdot g_{*}\left(\left[i d_{S^{1}}\right]\right)=$ $f_{*}(\alpha) \cdot[g]$ in $\pi_{1}(X)$ for any $\alpha \in \pi_{1}(U)$. Observe that $G_{1}(U, X ; f)$ is contained in the center of the fundamental group $\pi_{1}(X)$ if the induced map $f_{*}: \pi_{1}(U) \rightarrow \pi_{1}(X)$ is surjective. In particular the Gottlieb group $G_{1}(X)$ is abelian.

We further give a computational example (Theorem 1.9 below) whose proof illustrates how the elaborate machinery in this paper is relevant in computing Gottlieb groups. Consider the $S^{1}$-bundle $S^{1} \rightarrow X_{f} \rightarrow T^{n}$ over the $n$-dimensional torus $T^{n}$ with the classifying map $f$ which is represented by $\rho_{f}=\sum_{i<j} c_{i j} t_{i} t_{j}$ 
in $H^{2}\left(T^{n} ; \mathbb{Z}\right) \cong\left[T^{n}, K(\mathbb{Z}, 2)\right]$. Here $\left\{t_{i}\right\}_{1 \leq i \leq n}$ is a basis of $H^{1}\left(T^{n} ; \mathbb{Z}\right)$. Define an $(n \times n)$-matrix $A_{f}$ by $A_{f}=\left(c_{i j}^{\prime}\right)$, where $c_{i j}^{\prime}=c_{i j}$ for $i<j, c_{i j}^{\prime}=-c_{j i}$ for $i>j$ and $c_{i i}=0$. We regard $A_{f}$ as a matrix with entries in $\mathbb{Q}$. Then the rank of $A_{f}$ is denoted by $\operatorname{rank} A_{f}$. We establish the following theorem.

Theorem 1.9. $G_{1}\left(X_{f}\right) \cong \mathbb{Z}^{\oplus\left(1+n-\operatorname{rank} A_{f}\right)}$.

Since the space $X_{f}$ is aspherical, it follows from [12, Corollary I.13] that $G_{1}\left(X_{f}\right)$ coincides with the center of $\pi_{1}\left(X_{f}\right)$. While we have the central extension

$$
0 \rightarrow \mathbb{Z} \rightarrow \pi_{1}\left(X_{f}\right) \rightarrow \mathbb{Z}^{\oplus n} \rightarrow 0
$$

from the homotopy exact sequence of the fibration $S^{1} \rightarrow X_{f} \rightarrow T^{n}$, in general, it is not easy to determine the center of $\pi_{1}\left(X_{f}\right)$ by looking at the extension.

The rest of this paper is organized as follows. In Section 2, we recall the rational model for a function space constructed by Brown and Szczarba. We present an explicit model for a connected component of a function space in Section 3. In Section 4 , after introducing the notion of a detective element, we prove Theorem 1.1 by applying the model in Section 3; see Theorem 4.2. The main goal of Section 5 is to proving Theorem 1.2. In Section 6, we deal with the relationship between detective elements and higher order Whitehead products mentioned above. In Section 7, Theorem 1.6 is proved. We also give examples of separable and non-separable fibrations. Section 8 is devoted to proving Theorems 1.7 and 1.9. Moreover the first rational Gottlieb group of a non-aspherical space is computed; see Example 8.5.

We conclude this section with remarks on models for a function space. One might expect Haefliger's model [15] for the connected component of a function space to work well in considering the evaluation subgroups or, more generally, the homotopy type of $\mathcal{F}(U, X ; f)$. However it seems that the differential of the model is complicated in general because of the inductive argument in defining it. On the other hand, the model due to Brown and Szczarba has the advantage that its differential is expressed with an explicit formula; see (2.1) in Section 2. This is the reason why we draw on the latter in our study on evaluation subgroups. We also wish to mention that the two models above coincide before minimization, if the function space considered is connected; see [21, Theorem 1.1].

We are convinced that both our explicit model and derivations on Sullivan models used in [25], [26] and [27] are useful tools for the study of rational evaluation subgroups.

\section{AN ALGEBRAiC MODEl FOR A PATH COMPONENT OF A FUNCTION SPACE}

In this section, we summarize how to construct the Brown and Szczarba models for a function space and its connected component. Moreover, some fundamental properties of the models are described.

We emphasize that detailed knowledge of the model, in particular of the notation explained here is absolutely crucial to understanding the proofs later in the paper.

For a graded vector space $V$ over $\mathbb{Q}$, the free algebra generated by $V$ is denoted by $\wedge V$. The degree of a homogeneous element $v \in V$ is denoted by $\operatorname{deg} v$ or $|v|$. We shall use the terminology for algebraic models as used in [9]. Let $\left(A, d_{A}\right)$ be a connected differential free graded algebra, say $A=\wedge V$ for some graded vector space $V$. Let $\left(B, d_{B}\right)$ be a connected differential graded algebra (DGA) of finite 
type and $B_{*}$ denote the differential graded coalgebra defined by $B_{q}=\operatorname{Hom}\left(B^{-q}, \mathbb{Q}\right)$ for $q \leq 0$ together with the coproduct $D$ and the differential $d_{B *}$ which are dual to the multiplication of $B$ and to the differential $d_{B}$, respectively. Assume that $B$ is of finite type; that is, the vector space $B^{q}$ is finite dimensional for all $q$. Let $I$ be the ideal of the free algebra $\wedge\left(\wedge V \otimes B_{*}\right)$ generated by $1 \otimes 1-1$ and all elements of the form

$$
a_{1} a_{2} \otimes e-\sum_{i}(-1)^{\left|a_{2}\right|\left|e_{i}^{\prime}\right|}\left(a_{1} \otimes e_{i}^{\prime}\right)\left(a_{2} \otimes e_{i}^{\prime \prime}\right),
$$

where $a_{1}, a_{2} \in \wedge V, e \in B_{*}$ and $D(e)=\sum_{i} e_{i}^{\prime} \otimes e_{i}^{\prime \prime}$. Observe that $\wedge\left(\wedge V \otimes B_{*}\right)$ is a DGA with the differential $d:=d_{A} \otimes 1 \pm 1 \otimes d_{B *}$.

Theorem 2.1. [5, Theorems 3.3 and 3.5] (i) $\left(d_{A} \otimes 1 \pm 1 \otimes d_{B *}\right)(I) \subset I$.

(ii) The composite map

$$
\zeta: \wedge\left(V \otimes B_{*}\right) \hookrightarrow \wedge\left(\wedge V \otimes B_{*}\right) \rightarrow \wedge\left(\wedge V \otimes B_{*}\right) / I
$$

is an isomorphism of graded algebras.

This theorem enables us to define a differential $\delta$ on $\wedge\left(V \otimes B_{*}\right)$ by $\zeta^{-1} \tilde{d} \zeta$, where $\tilde{d}$ is the differential on $\wedge\left(\wedge V \otimes B_{*}\right) / I$ induced by $d$. Let $D^{(m-1)}: B_{*} \rightarrow B_{*}^{\otimes m}$ be the iterated coproduct on $B_{*}$. For an element $v \in V$ and a cycle $e \in B_{*}$, if $d(v)=v_{1} \cdots v_{m}$ with $v_{i} \in V$ and $D^{(m-1)}(e)=\sum_{j} e_{j_{1}} \otimes \cdots \otimes e_{j_{m}}$, then

$$
\delta(v \otimes e)=\sum_{j} \pm\left(v_{1} \otimes e_{j_{1}}\right) \cdots\left(v_{m} \otimes e_{j_{m}}\right),
$$

where the sign is determined by the Koszul rule that in a graded algebra $a b=$ $(-1)^{\operatorname{deg} a \operatorname{deg} b} b a$. It follows from $[5$, Lemma 5.1] that if $(\wedge V, d)$ is a Sullivan algebra, then so is $\left(\wedge\left(V \otimes B_{*}\right), \delta\right)$.

Let $\Delta[q]$ be the simplicial set consisting of non-decreasing maps to the ordered set $[q]=\{0,1, \ldots, q\}$. As usual we can write

$$
\Delta[q]_{p}=\left\{\left(i_{0}, i_{1}, \ldots, i_{p}\right) \mid 0 \leq i_{0} \leq \cdots \leq i_{p} \leq q\right\} .
$$

Let $\Delta \mathcal{S}$ be the category of simplicial sets. For $K, L \in \Delta \mathcal{S}$, let $\operatorname{Simpl}(K, L)$ stand for the set of simplicial maps from $K$ to $L$. The function space $\mathcal{F}(K, L) \in \Delta \mathcal{S}$ is defined by

$$
\mathcal{F}(K, L)_{q}=\operatorname{Simpl}(K \times \Delta[q], L) .
$$

Let $A_{P L}$ be the simplicial commutative cochain algebra of polynomial differential forms with coefficients in $\mathbb{Q}$; see [2] and [9, section 10]. Let $\mathcal{A}$ be the category of DGA's over $\mathbb{Q}$. For $A, B \in \mathcal{A}$, let $\operatorname{DGA}(A, B)$ denote the set of DGA maps from $A$ to $B$. Following Bousfield and Gugenheim [2], we define functors

$$
\Delta: \mathcal{A} \rightarrow \Delta \mathcal{S} \text { and } \Omega: \Delta \mathcal{S} \rightarrow \mathcal{A}
$$

by $\Delta(A)=\operatorname{DGA}\left(A, A_{P L}\right)$ and by $\Omega(K)=\operatorname{Simpl}\left(K, A_{P L}\right)$, respectively. For any objects $A$ and $B$ in $\mathcal{A}$, we define the function space $\mathcal{F}(A, B) \in \Delta \mathcal{S}$ by

$$
\mathcal{F}(A, B)_{q}=\operatorname{DGA}\left(A,\left(A_{P L}\right)_{q} \otimes B\right) .
$$

The singular simplicial set is denoted by $\Delta U$ for any topological space $U$ and $|K|$ denotes the geometric realization of a simplicial set $K$. We refer to the space $|\Delta(A)|$ as the Sullivan realization of $A$. Observe that the differential graded algebra $A_{P L}(U)$ of rational polynomial differential forms on $U$ is then given by $A_{P L}(U)=$ $\Omega \Delta U$. For any $K, L \in \Delta \mathcal{S}$, we define a map of simplicial sets

$$
\alpha: \mathcal{F}(K, L) \rightarrow \Delta \mathcal{F}(|K|,|L|)
$$


by $\alpha(f)=|f|:|K \times \Delta[q]| \rightarrow|L|$ for $f \in \mathcal{F}(K, L)_{q}$. For any space $U$, let $s:|\Delta U| \rightarrow$ $U$ denote the homotopy equivalence defined by $s(\sigma, f)=f(\sigma)$; see, for example, [6, (12.10)]. There exists a sequence of homotopy equivalences

$$
\mathcal{F}(U, X) \simeq \mathcal{F}(|\Delta U|,|\Delta X|) \stackrel{s}{\simeq}|\Delta \mathcal{F}(|\Delta U|,|\Delta X|)| \stackrel{|\alpha|}{\simeq}|\mathcal{F}(\Delta U, \Delta X)|
$$

for any topological spaces $U$ and $X$; see [5, Theorem 2.1].

Let $m: \wedge V=A \stackrel{\simeq}{\longrightarrow} \Omega \Delta X$ be the minimal model for $\Delta X$ and $\beta: B \stackrel{\simeq}{\longrightarrow} \Omega \Delta U$ a quasi-isomorphism in which $B$ is of finite type but not necessarily free as a graded algebra. For any simplicial set $K$, we can define a bijection

$$
\eta: \operatorname{DGA}(A, \Omega(K)) \longrightarrow \operatorname{Simpl}(K, \Delta(A))
$$

by $\eta: \phi \mapsto f ; f(\sigma)(a)=\phi(a)(\sigma)$, where $a \in A$ and $\sigma \in K_{n}$. The map $m$ : $A \stackrel{\simeq}{\longrightarrow} \Omega \Delta X$ induces a $\mathbb{Q}$-localization $h: \Delta X \rightarrow \Delta(A)$ via the bijection $\eta$ if $X$ is a connected nilpotent space; see [2], [9, Theorem 17.12].

Remark 2.2. Suppose that $U$ is a connected finite CW complex and $X$ is a connected nilpotent space. Then it follows from [19, Theorem 3.11] that the map $h$ induces a Q-localization

$$
h_{*}: \mathcal{F}(\Delta U, \Delta X) \longrightarrow \mathcal{F}(\Delta U, \Delta A) .
$$

Let $\left\{b_{i}\right\}$ be a basis for $B$ and $\left\{e_{i}\right\}$ its dual basis for $B_{*}$. For differential graded algebras $C$ and $D$, let $\operatorname{DGM}(C, D)$ denote the set of morphisms from $C$ to $D$ in the category of differential graded $\mathbb{Q}$-vector spaces. Define the map $\Psi: \operatorname{DGM}(A \otimes$ $\left.B_{*}, A_{P L}\right) \rightarrow \operatorname{DGM}\left(A, A_{P L} \otimes B\right)$ in $\Delta \mathcal{S}$ by

$$
\Psi(w)(a)=\sum_{i}(-1)^{\mu\left(\left|b_{i}\right|\right)} w\left(a \otimes e_{i}\right) \otimes b_{i},
$$

where $\mu(n)=[(n+1) / 2]$, the greatest integer in $(n+1) / 2$. Then the map induces a simplicial isomorphism $\Psi: \Delta\left(\wedge\left(A \otimes B_{*}\right) / I, \widetilde{d}\right) \rightarrow \mathcal{F}(A, B)$ ([5, Corollary 3.4]). Moreover, we have a sequence consisting of the simplicial isomorphism $\Psi$ and homotopy equivalences

$$
\mathcal{F}(\Delta U, \Delta A) \stackrel{\widetilde{\eta}}{\simeq} \mathcal{F}(A, \Omega \Delta U) \underset{\simeq}{\stackrel{\beta_{*}}{\simeq}} \mathcal{F}(A, B) \underset{\cong}{\stackrel{\Psi}{\cong}} \Delta\left(\wedge\left(A \otimes B_{*}\right) / I, \widetilde{d}\right) .
$$

Observe that the homotopy equivalence $\tilde{\eta}$ is induced by the quasi-isomorphism $\Omega \Delta U \otimes\left(A_{P L}\right)_{q} \cong \Omega \Delta U \otimes \Omega(\Delta[q]) \rightarrow \Omega(\Delta U \times \Delta[q])$ and the bijection $\eta$; see [4, Theorem 1.29]. For a simplicial set $K$, define $\xi_{K}: K \rightarrow \Delta|K|$ by $\xi_{K}(\sigma)=t_{\sigma}$ : $\Delta^{n} \rightarrow\{\sigma\} \times \Delta^{n} \rightarrow|K|$. We have a sequence of DGA maps

$$
\begin{aligned}
\left.\Omega \Delta \mid \Delta\left(\wedge\left(A \otimes B_{*}\right) / I, \widetilde{d}\right)\right) \mid \stackrel{\Omega\left(\xi_{K}\right)}{\longrightarrow} \Omega \Delta\left(\wedge\left(A \otimes B_{*}\right) / I, \widetilde{d}\right) \stackrel{\Omega \Delta \zeta}{\stackrel{\Omega}{\longrightarrow}} \Omega\left(\wedge\left(V \otimes B_{*}\right), \delta\right) \\
\eta^{-1}(i d) \uparrow \\
\left(\wedge\left(V \otimes B_{*}\right), \delta\right)
\end{aligned}
$$

in which $\Omega\left(\xi_{K}\right)$ and $\Omega \Delta \zeta$ are quasi-isomorphism and $\eta^{-1}(i d)$ denotes the adjunction map to the identity map $i d$ on the simplicial set $\Delta\left(\left(\wedge\left(V \otimes B_{*}\right), \delta\right)\right)$. Applying the realization functor $|\quad|$ and the functor $A_{P L}(\quad)$ to the sequence (2.3), and by combining the resultant sequence with the above sequence, we obtain quasi-isomorphisms which connect $A_{P L}(\mathcal{F}(U, X))=\Omega \Delta(\mathcal{F}(U, X))$ with $\Omega \Delta\left(\wedge\left(V \otimes B_{*}\right), \delta\right)$.

A minimal model $E=\wedge W$ of $\wedge\left(V \otimes B_{*}\right)$ is constructed as follows: Let $\left\{a_{k}, b_{k}, c_{j}\right\}_{k, j}$ be a basis for $B_{*}$ such that $d_{B_{*}}\left(a_{k}\right)=b_{k}$ and $d_{B_{*}}\left(c_{j}\right)=0$. Without loss of generality, we can assume that $c_{0}=1$. Choose a basis $\left\{v_{i}\right\}_{i \geq 1}$ for $V$ so that 
$\operatorname{deg} v_{i} \leq \operatorname{deg} v_{i+1}$ and $d\left(v_{i+1}\right) \in \wedge V_{i}$, where $V_{i}$ is the subspace spanned by the elements $v_{1}, \ldots, v_{i}$. The result [5, Lemma 5.1] states that there exist free algebra generators $w_{i j}, u_{i k}$ and $v_{i k}$ such that

(2.4) $w_{i 0}=v_{i} \otimes 1$ and $w_{i j}=v_{i} \otimes c_{j}+x_{i j}$, where $x_{i j} \in \wedge\left(V_{i-1} \otimes B_{*}\right)$,

(2.5) $\delta w_{i j}$ is decomposable and in $\wedge\left(\left\{w_{s l} ; s<i\right\}\right)$,

(2.6) $u_{i k}=v_{i} \otimes a_{k}$ and $\delta u_{i k}=v_{i k}$.

We then have a decomposition

$$
\wedge\left(V \otimes B_{*}\right)=\wedge\left(w_{i j}\right)_{i, j} \otimes \wedge\left(u_{i k}, v_{i k}\right)_{i, k}
$$

in the category of DGA's. It follows that the inclusion

$$
\gamma: E:=\left(\wedge\left(w_{i j}\right), \delta\right) \hookrightarrow\left(\wedge\left(V \otimes B_{*}\right), \delta\right)
$$

is a homotopy equivalence whose inverse is the projection

$$
\rho:\left(\wedge\left(V \otimes B_{*}\right), \delta\right) \rightarrow E
$$

see [5, Lemma 5.2] for example.

It follows from (2.4) that the vector space generated by the elements $w_{i j}$ is isomorphic to $V \otimes H_{*}\left(B_{*}\right)$ as a vector space. Thus we have $E \cong \wedge\left(V \otimes H_{*}(U)\right)$. In consequence, we see that $\Delta(E)$ is homotopy equivalent to $\Delta\left(\wedge\left(V \otimes B_{*}\right), \delta\right)$, and hence to the function space $\mathcal{F}(\Delta U, \Delta A)$.

In what follows, we assume that

$$
\operatorname{dim} \oplus_{q \geq 0} H^{q}(U ; \mathbb{Q})<\infty \text { or } \operatorname{dim} \oplus_{i \geq 2} \pi_{i}(X) \otimes \mathbb{Q}<\infty .
$$

We shall describe a model for a connected component of a function space. Let $K$ be a simplicial set and $u$ an element in $K_{0}$. We say that an element $x \in K_{s}$ has a vertex $u$ if $d_{i_{1}} \cdots d_{i_{s}} x=u$ for any $i_{1}, \ldots, i_{s}$. Let $\Delta(E)_{u}$ denote the connected component of $u \in \Delta(E)_{0}$; that is, the simplicial subset of $\Delta(E)$ consisting of all elements all of whose vertices are at $u$. Let $M_{u}$ be the ideal of $E$ generated by the set

$$
\{\omega \mid \operatorname{deg} \omega<0\} \cup\{\delta \omega \mid \operatorname{deg} \omega=0\} \cup\{\omega-u(\omega) \mid \operatorname{deg} \omega=0\} .
$$

Theorem 2.3. [5, Theorem 6.1]. The ideal $M_{u}$ is closed under the differential $\delta$ and the quotient map $\pi: E \rightarrow E / M_{u}$ induces a homotopy equivalence $\Delta(\pi): \Delta\left(E / M_{u}\right) \rightarrow \Delta(E)_{u}$. Moreover $\left(E / M_{u}, \delta\right)$ is a Sullivan algebra, which is not necessarily minimal, and is isomorphic to $\wedge(\widetilde{W})$, where $\widetilde{W}^{q}=0$ for $q<1$, $\widetilde{W}^{1} \subset W^{1}$ and $\widetilde{W^{q}}=W^{q}$ for $q>1$.

By forming the quotient $E / M_{u}$, one eliminates all elements of negative degree. Moreover an element $\omega$ of degree 0 becomes a cycle, identified with the scalar $u(\omega)$.

We choose an element $u \in \Delta(E)_{0}$. Let $\varepsilon: B \rightarrow \mathbb{Q}$ be the augmentation of $B$. The sequence of simplicial sets and simplicial maps

$$
\Delta\left(E / M_{u}\right) \stackrel{\Delta(\pi)}{\simeq} \Delta(E)_{u} \underbrace{\longrightarrow}_{\Delta(\pi)} \Delta(E) \stackrel{\Delta(\rho)}{\simeq} \Delta\left(\wedge\left(A \otimes B_{*}\right) / I\right) \stackrel{\Delta\left(1 \otimes \varepsilon^{\sharp}\right)}{\longrightarrow} \Delta(\wedge V)
$$


gives rise to a commutative diagram

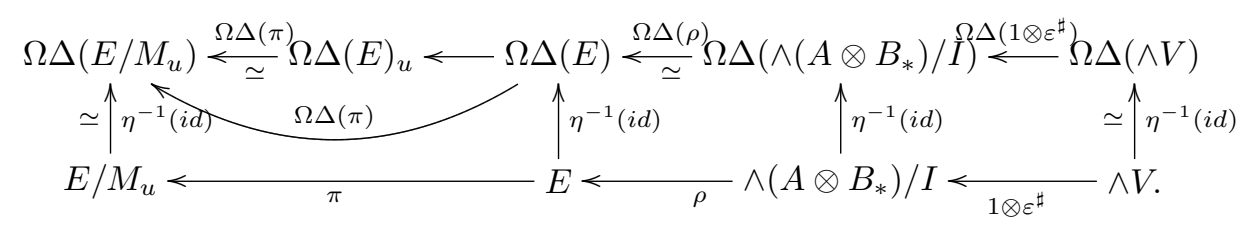

It follows from the hypothesis (2.9) that $E / M_{u}$ is of finite type and hence so is the homology of $E / M_{u}$. Therefore the result [2, 10.1 Theorem] yields that the adjoint $\eta^{-1}(i d): E / M_{u} \rightarrow \Omega \Delta\left(E / M_{u}\right)$ is a quasi-isomorphism.

We can regard the map $\Delta\left(1 \otimes \varepsilon^{\sharp}\right)$ as the morphism $\mathcal{F}(\Delta(j), 1): \mathcal{F}(\Delta U, \Delta A) \rightarrow$ $\mathcal{F}(\Delta(*), \Delta A)$ of simplicial sets induced by the natural inclusion $j: * \rightarrow U$ up to the homotopy equivalences in (2.3); see [21, Appendix]. Moreover the realization of $\mathcal{F}(\Delta(j), 1)$ is nothing but the evaluation map ev $: \mathcal{F}(U,|\Delta A|) \rightarrow|\Delta A|$. Let $\delta_{0}$ denote the linear part of the differential $\delta$ of $\wedge(\widetilde{W})=E / M_{u}$. Write $\widetilde{W}=$ $C \oplus \delta_{0} C \oplus H\left(\widetilde{W}, \delta_{0}\right)$ with an appropriate subspace $C$ of $\widetilde{W}$ and put $\bar{W}=H\left(\widetilde{W}, \delta_{0}\right)$. As usual, we can construct a minimal model $n: \wedge \bar{W} \rightarrow E / M_{u}=\wedge(C \oplus \delta C \oplus \bar{W})$ together with the retraction $r: E / M_{u} \rightarrow \wedge(\bar{W})$, which is defined by extending the projection $C \oplus \delta C \oplus \bar{W} \rightarrow \bar{W}$. It is readily seen that

$$
r \pi \rho\left(1 \otimes \varepsilon^{\sharp}\right): \wedge V \rightarrow \wedge\left(A \otimes B_{*}\right) / I \rightarrow E \rightarrow E / M_{u} \rightarrow \wedge \bar{W}
$$

is a Sullivan representative for the evaluation map ev $: \mathcal{F}\left(U, X_{\mathbb{Q}} ; f\right) \rightarrow X_{\mathbb{Q}}$, where $X_{\mathbb{Q}}=|\Delta(A)|$ and $f$ is the map corresponding to the element $\left(0, \tilde{\eta} \beta_{*} \Psi \Delta(\rho)(u)\right) \in$ $|\mathcal{F}(\Delta U, \Delta(A))|$ by the homotopy equivalence $s \circ|\alpha|$, see $(2.2)$.

Remark 2.4. Let $\tilde{u}$ be a 0 -simplex in $\Delta\left(\wedge\left(A \otimes B_{*}\right) / I\right)$ and put $u=\Delta(\gamma)(\widetilde{u})$. Since $\Delta(\rho) \Delta(\gamma) \simeq i d$, it follows that there exists a path connecting $f=(s \circ$ $|\alpha|)\left(0, \tilde{\eta} \beta_{*} \Psi \Delta(\rho)(u)\right)$ with $g=(s \circ|\alpha|)\left(0, \tilde{\eta} \beta_{*} \Psi(\widetilde{u})\right)$. Hence we have $\mathcal{F}\left(U, X_{\mathbb{Q}} ; f\right)=$ $\mathcal{F}\left(U, X_{\mathbb{Q}} ; g\right)$

The following proposition clarifies a property of the minimal model $(\wedge \bar{W}, \delta)$ for the function space $\mathcal{F}\left(U, X_{\mathbb{Q}} ; f\right)$, which is deduced from knowledge of the differential of a minimal model for $X$ independently of any property of a model for $U$.

Proposition 2.5. Suppose that linearly independent elements $u_{1}, \ldots, u_{s}$ in $V$ do not occur in any terms of the images of the differential $d: V \rightarrow \wedge V$ as factors. Then the elements $u_{1} \otimes 1, \ldots, u_{s} \otimes 1$ are linearly independent in $\bar{W}=H\left(\widetilde{W}, \delta_{0}\right) \subset E / M_{u}$.

Proof. Let $z$ be a non-trivial linear combination of elements $u_{1}, \ldots, u_{s}$. It is immediate that $\delta_{0}(z \otimes 1)=0$ since $d z$ is decomposable. In order to prove the proposition, it suffices to show that $z \otimes 1$ does not occur in any terms of the images of $\delta_{0}$ as a factor. For that purpose, we choose elements $w_{i j}(j>0)$ satisfying $(2.4)$ and $(2.5)$ as follows: Let $\left\{v_{i}\right\}_{i}$ be a basis which contains the element $z$. We argue by induction on lower degree $i$ of the base elements $v_{i}$ of $V$. By assumption, the element $z$ does not occur in any term of the images of $d: V \rightarrow \wedge V$. Thus we have a sub DGA of the form

$$
\left(\wedge\left(v_{k} \otimes e ; k<i, e \in\left\{a_{m}, b_{m}, c_{n}\right\}, v_{k} \otimes e \neq z \otimes 1\right), \delta\right)
$$


of $\left(\wedge\left(V_{i-1} \otimes B_{*}\right), \delta\right)$, where $\left\{a_{m}, b_{m}, c_{n}\right\}$ is a basis for $B_{*}$ mentioned above. Then we see that

$$
\begin{aligned}
{\left[\delta\left(v_{i} \otimes c_{j}\right)\right] } & \in H\left(\wedge\left(v_{k} \otimes e ; k<i, e \in\left\{a_{m}, b_{m}, c_{n}\right\}, v_{k} \otimes e \neq z \otimes 1\right), \delta\right) \\
& \cong H\left(\wedge\left(w_{l n} ; l<i, w_{l n} \neq z \otimes 1\right) \otimes \wedge\left(u_{l m}, v_{l m} ; l<i\right)\right) \\
& \cong H\left(\wedge\left(w_{l n} ; l<i, w_{l n} \neq z \otimes 1\right)\right) .
\end{aligned}
$$

It follows that there exists $x_{i j} \in \wedge\left(v_{k} \otimes e ; k<i, v_{k} \otimes e \neq z \otimes 1\right)$ such that $\delta\left(v_{i} \otimes c_{j}\right)+$ $\delta x_{i j} \in \wedge\left(w_{l n} ; l<i, w_{l n} \neq z \otimes 1\right)$. It is evident that the element $w_{i j}=v_{i} \otimes c_{j}+x_{i j}$ satisfies the conditions (2.4) and (2.5). We will prove this proposition with the elements $w_{i j}(j>0)$ and $w_{i 0}$, which generate the algebra $E$ mentioned above.

Suppose that $\delta(\pi y)=z \otimes 1+w$ for some $y \in E \subset \wedge\left(A \otimes B_{*}\right) / I$, where $w$ is an element of $E / M_{u}$ which does not have a term containing $z \otimes 1$ as a factor. Then we write

$$
w+z \otimes 1=\pi \delta\left(\sum_{i_{l}, j_{l}} w_{i_{1} j_{1}} \cdots w_{i_{s} j_{s}}\right),
$$

which is a contradiction because each $\pi \delta w_{i_{l} j_{l}}$ does not have a term containing $z \otimes 1$ as a summand. This completes the proof.

Remark 2.6. Let $z \in V$ be a non-zero element and $\left\{v_{i}\right\}_{i}$ a basis of $V$ which contains $z$. The proof of Proposition 2.5 yields that, if $z \otimes 1 \in \operatorname{Im} \delta_{0}$, then there exists an element $v_{l} \in\left\{v_{i}\right\}_{i}$ such that $\delta\left(v_{l} \otimes e\right)$ has a term containing $z \otimes 1$ as a summand for some $e \in B_{*}$. This implies that some term of $d v_{l}$ contains the element $z$ as a factor.

\section{An EXPLiCit MODEL FOR $\mathcal{F}(U, X ; f)$}

In this section, we assume that $U$ and $X$ are a connected nilpotent space and a connected rational space, respectively, and that the rational homologies of $U$ and $X$ are of finite type. Moreover it is assumed that the condition (2.9) holds for $U$ and $X$.

Let $f: U \rightarrow X$ be a map and $(\wedge V, d)$ a minimal model for $X$. We take a Sullivan model $i:(\wedge V, d) \longmapsto(\wedge V \otimes \wedge Z, \widehat{d})$ for $f$. Consider a minimal model $\varphi: \wedge W \cong \wedge V \otimes \wedge Z$ and a lift $q: \wedge V \rightarrow \wedge W$ of the model $i$ (see [9, Proposition 14.6]). We then have a diagram

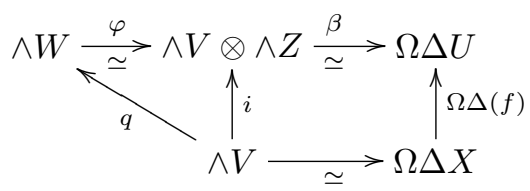

in which the right square is commutative and the left triangle is commutative up to homotopy. Observe that the composition $\theta:=\beta \varphi: \wedge W \rightarrow \Omega \Delta U$ is a minimal model for $U$.

In order to construct an explicit model for $\mathcal{F}(U, X ; f)$ using the model $i$ for the given map $f$, we have to verify the finiteness of $\wedge V \otimes \wedge Z$.

Lemma 3.1. In the minimal Sullivan model for $f$ mentioned above, the algebra $\wedge V \otimes \wedge Z$ is of finite type.

Proof. We observe that $\wedge V$ and $\wedge W$ are of finite type. Since $\widehat{d}_{0}$ sends $Z$ to $V$, it follows that $\pi^{*}(\wedge W) \cong \pi^{*}(\wedge V \otimes \wedge Z)=\left(V / \widehat{d}_{0}(Z)\right) \oplus \operatorname{ker} \widehat{d_{0}}$. The minimality of $\wedge W$ 
implies that $\pi^{*}(\wedge W)=W$. We write $Z=\operatorname{ker} \widehat{d}_{0} \oplus T$ with a subspace $T$ for which $\widehat{d}_{0}: T \rightarrow \widehat{d}_{0}(Z)$ is an isomorphism. This completes the proof.

Lemma 3.2. In the homotopy set $[U, X], f=|\Delta(i) \eta(\beta)|$.

Proof. Since $X$ is regarded as the realization $|\Delta A|$, we see that $f=|\Delta(q) \eta(\theta)|$ in $[U, X]$. The naturality of the map $\eta$ allows us to conclude that $\Delta(q) \eta(\theta)=\eta(\theta q)=$ $\eta(\beta i)=\Delta(i) \eta(\beta)$. Observe that all equalities are of homotopy classes.

Put $B=\wedge V \otimes \wedge Z$. With the notation in the previous section, define $\tilde{u} \in$ $\operatorname{DGM}\left(\wedge V \otimes B_{*},\left(A_{P L}\right)_{0}\right)$ by

$$
\tilde{u}(a \otimes e)=(-1)^{\mu(|a|)} e(i(a)),
$$

where $a \in \wedge V$ and $e \in B_{*}$. Then we see that $\Psi(\tilde{u})=i \in \mathcal{F}(\wedge V, B)_{0}$. The result [5, Theorem 3.3] enables us to conclude that $\tilde{u}$ is a 0 -simplex of $\Delta\left(\wedge\left(\wedge V \otimes B_{*}\right) / I\right)$; that is $\widetilde{u}$ is a morphism of algebras from $\wedge\left(\wedge V \otimes B_{*}\right) / I$ into $\left(A_{P L}\right)_{0}$. By the straightforward calculation, we can verify that $\tilde{\eta} \beta_{*} \Psi(\widetilde{u})=\Delta(i) \eta(\beta) \in \mathcal{F}(\Delta U, \Delta(\wedge V))_{0}$. Moreover it follows from Lemma 3.2 that $(s \circ|\alpha|)(0, \Delta(i) \eta(\beta))=|\Delta(i) \eta(\beta)|=$ $f \in \mathcal{F}(U,|\Delta(\wedge V)|)$. Recall the inclusion $\gamma:(E, \delta) \rightarrow\left(\wedge\left(V \otimes B_{*}\right), \delta\right)$ which is a homotopy equivalence; see (2.7). We establish the following theorem.

Theorem 3.3. Under the hypothesis (2.9), the differential graded algebra $E / M_{u}$ is a Sullivan model for the function space $\mathcal{F}(U, X ; f)$, where $u=\Delta(\gamma) \tilde{u}$. Moreover the map

$$
\iota:(\wedge V, d) \rightarrow\left(E / M_{u}, \delta\right)
$$

defined by $\iota(v)=v \otimes 1$ is a model for the evaluation map ev $: \mathcal{F}(U, X ; f) \rightarrow X$.

Proof. The result follows from Theorem 2.3, the ensuing discussion and Remark 2.4 .

For any 0-simplex $u \in \Delta(E)_{0}$, a model of the connected component of the function space $\mathcal{F}(|\Delta(\wedge V)|,|\Delta U|)$ containing the map $\left|\widetilde{\eta} \beta_{*} \Psi \Delta(\rho) u\right|:|\Delta(\wedge V)| \rightarrow$ $|\Delta U|$, which corresponds to $u$, is given in [5]; see (2.3) and (2.10) for notations. We emphasize that Theorem 3.3 gives not only an explicit model for the connected component $\mathcal{F}(U, X ; f)$ containing a given map $f$, but also a rational model for the evaluation map in terms of the Brown-Szczarba model.

By using the models in Theorem 3.3 for the function space $\mathcal{F}(U, X ; f)$ and for the evaluation map, we will prove Theorem 1.1 in the next section.

Remark 3.4. We choose the Sullivan representative $q: \wedge V \rightarrow \wedge W$ for $f$ instead of the Sullivan model $i$. Observe that $\wedge V$ and $\wedge W$ are of finite type because $U$ and $X$ are nilpotent. By the same argument as above, we can construct a model of the form $E / M_{u}$ for $\mathcal{F}(U, X ; f)$ by choosing $\wedge W$ as a model for $U$ instead of $\wedge V \otimes \wedge Z$. In this case, the 0 -simplex $\widetilde{u} \in \Delta\left(\wedge\left(\wedge V \otimes B_{*}\right) / I\right)$, which corresponds to $f$ under homotopy equivalences in (2.2) and (2.3), is defined by

$$
\widetilde{u}_{q}(a \otimes e)=(-1)^{\mu(|a|)} e(q(a)) .
$$

It turns out that Theorem 3.3 remains valid if $B=\wedge W$ and the 0 -simplex $u$ is replaced by $\Delta(\gamma) \widetilde{u}_{q}$.

It seems that this model for computing the evaluation subgroup $G_{*}(U, X ; f)$ works well when a Sullivan representative of $f$ is comparatively tractable. 
The following lemma is useful for determining whether an element of the rational homotopy group of a space is in an evaluation subgroup; see Example 3.6 below.

Lemma 3.5. Let $\iota: \wedge V \rightarrow E / M_{u}=\wedge \widetilde{W}$ be the model for ev in Theorem 3.3 or Remark 3.4 and $n: \wedge \bar{W} \rightarrow E / M_{u}$ the minimal model with the retraction $r$ described before Remark 2.4, where $\bar{W}=H^{*}\left(\widetilde{W}, \delta_{0}\right)$. Put $\widetilde{e v}=r \circ \iota$ and let $Q(\widetilde{e v})$ be the linear part of $\widetilde{e v}$. Then $Q(\widetilde{e v})(v)=0$ for an element $v \in V$ if and only if $\iota(v)$ is in $\operatorname{Im}\left\{\delta: \widetilde{W} \rightarrow E / M_{u}\right\}$ modulo decomposable elements, where $\delta$ denotes the differential on $E / M_{u}$.

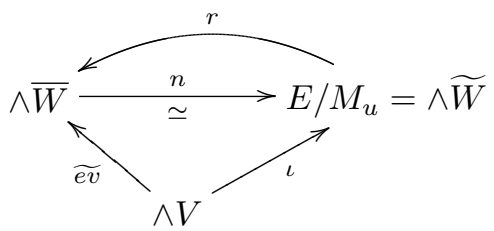

Proof. Observe that the retraction $r$ is defined by extending the projection $C \oplus \delta C \oplus$ $H^{*}\left(\widetilde{W}, \delta_{0}\right) \rightarrow \bar{W}$. Let $\pi^{\prime}: \wedge \bar{W} \rightarrow \bar{W} \cong \wedge \bar{W} /(\bar{W} \cdot \bar{W})$ be the projection. Suppose that, for an element $v \in V, Q(\widetilde{e v})(v)=0$. Since $v \otimes 1 \in \operatorname{Ker} \delta_{0}$, it follows that $v \otimes 1 \in \delta C \oplus \bar{W}$ modulo decomposable elements. Therefore we see that $v \otimes 1 \in \delta C$ modulo decomposable elements since $Q(\widetilde{e v})(v)=\pi^{\prime} r(v \otimes 1)$.

If $\delta(\alpha) \equiv v \otimes 1$ for some $\alpha \in C \oplus \delta C \oplus \bar{W}$; that is, $\delta(\alpha)=v \otimes 1$ modulo decomposable elements, then there exists an element $\alpha^{\prime} \in C$ such that $\delta\left(\alpha^{\prime}\right) \equiv v \otimes 1$. We have $Q(\widetilde{e v})(v)=0$.

Example 3.6. Let $k$ be an positive integer. We define a DGA $\left(A_{k}, d\right)$ by $A_{k}=$ $\wedge V_{k}=\wedge\left(v, w_{k}\right)$ and $d\left(w_{k}\right)=v^{k+1}$. Let $q: A_{k} \rightarrow A_{l}$ be a DGA map, where $k \geq l$. Then it is readily seen that $q(v)=c v$ for some $c \in \mathbb{Q}$ and $q\left(w_{k}\right)=c^{k+1} v^{k-l} w_{l}$. Consider the Sullivan model $\left(E / M_{u}, \delta\right)$ for $\mathcal{F}\left(\left|\Delta\left(A_{l}\right)\right|,\left|\Delta\left(A_{k}\right)\right| ;|\Delta q|\right)$ described in Remark 3.4. Recall that $u(\omega)=\omega$ in $E / M_{u}$ for all $\omega$ of degree 0. Since $v \otimes v_{*}=$ $\tilde{u}\left(v \otimes v_{*}\right)=(-1)^{\mu(|v|)} v_{*}(q(v))$ and $v \otimes\left(v^{m}\right)_{*}=0$ for $m>1$, we see that

$$
\begin{aligned}
\delta\left(w_{k} \otimes\left(v^{s}\right)_{*}\right) & =d\left(w_{k}\right) \otimes\left(v^{s}\right)_{*}-w_{k} \otimes d_{*}\left(\left(v^{s}\right)_{*}\right) \\
& =v^{k+1} \cdot \frac{1}{s !}\left(D^{(k)}\left(v_{*}\right)\right)^{s} \quad(\operatorname{by}(2.1)) \\
& =\left((-1)^{\mu(|v|)} v_{*}(q(v))\right)^{s}\left(\begin{array}{c}
k+1 \\
s
\end{array}\right)\left(v \otimes 1_{*}\right)^{k+1-s} \\
& =(-1)^{s \mu(|v|)} c^{s}\left(\begin{array}{c}
k+1 \\
s
\end{array}\right)\left(v \otimes 1_{*}\right)^{k+1-s}
\end{aligned}
$$

where $s<k+1$. Observe that $s !\left(v^{s}\right)_{*}=\left(v_{*}\right)^{s}$ in the Hopf algebra $A_{k}^{\sharp}=\wedge\left(V_{k}^{\sharp}\right)$. In particular, we have $\delta\left(w_{k} \otimes\left(v^{k}\right)_{*}\right)=(-1)^{k \mu(|v|)} c^{k}(k+1) v \otimes 1_{*}$. Let $X_{k}$ be the spatial realization $\left|\Delta A_{k}\right|$ of $A_{k}$ and put $f=|\Delta q|$. As is mentioned before Remark 2.4 , the Sullivan model $\left(E / M_{u}, \delta\right)$ has a minimal model $(\wedge \bar{W}, \bar{\delta})$. Recall that

$$
G_{*}\left(X_{l}, X_{k} ; f\right)^{\sharp} \cong V_{k} / \operatorname{Ker} Q(\widetilde{e v}),
$$

where $\widetilde{e v}: A_{k} \rightarrow \wedge \bar{W}$ is the Sullivan representative of the evaluation map ev : $\mathcal{F}\left(X_{l}, X_{k} ; f\right) \rightarrow X_{k}$ as in Lemma 3.5. By virtue of Proposition 2.5, we see that $w_{k}$ is not in $\operatorname{Ker} Q(\widetilde{e v})$. Moreover the computation above and Lemma 3.5 allow us to conclude that $v$ is in $\operatorname{Ker} Q(\widetilde{e v})$ if $c \neq 0$. Thus we have $G_{*}\left(X_{l}, X_{k} ; f\right)=G_{*}\left(X_{k}\right)$ if 
$q \neq 0$ and $G_{*}\left(X_{l}, X_{k} ; f\right)=\pi_{*}\left(X_{k}\right)$ if $q=0$. This computation also implies that the Gottlieb group $G_{*}\left(X_{k}\right)$ is a proper subgroup of $\pi_{*}\left(X_{k}\right)$ if $q \neq 0$. Especially, we see that $G_{l}\left(\mathbb{C} P^{n}\right) \otimes \mathbb{Q}=\pi_{l}\left(\mathbb{C} P^{n}\right) \otimes \mathbb{Q}$ if and only if $l \neq 2$ and that $G_{2}\left(\mathbb{C} P^{n}\right) \otimes \mathbb{Q}=0$. Observe that $G_{*}(X) \otimes \mathbb{Q} \cong G_{*}\left(X_{\mathbb{Q}}\right)$ for a simply connected finite CW complex $X$; see [22, Corollary 2.5] and [35, Corollary 2.5].

We here describe a result which follows immediately from Proposition 2.5 and Lemma 3.5. Recall that a simply connected space is $X$ elliptic if $\operatorname{dim} H^{*}(X ; \mathbb{Q})<\infty$ and $\operatorname{dim} \pi_{*}(X) \otimes \mathbb{Q}<\infty$; see $[9, \S 32]$.

Theorem 3.7. (cf. [35, Theorem 4.1]) Let $U$ be a connected nilpotent space and $X$ a rationally nontrivial simply-connected space with only finitely many rational homotopy groups. Then for any $f: U \rightarrow X_{\mathbb{Q}}, G_{*}\left(U, X_{\mathbb{Q}} ; f\right) \neq 0$. In particular, $G_{*}\left(X_{\mathbb{Q}}\right) \neq 0$ if $X$ is rationally nontrivial and elliptic.

Proof. Let $(\wedge V, d)$ be a minimal model for $X$. We choose a nonzero element $v \in$ $V=\pi_{*}(X)^{\sharp}$ so that $\pi_{i}(X)=0$ for $i>\operatorname{deg} v$. It is evident that $v$ does not occur in any term of the differential $d$ as a factor. It follows from Proposition 2.5 that $\iota(v)=$ $v \otimes 1 \neq 0$ in $\bar{W}$. Lemma 3.5 yields that $Q(\widetilde{e v})(v) \neq 0$. Therefore $v$ is a non-zero element in $V / \operatorname{Ker} Q(\widetilde{e v}) \cong G_{*}\left(U, X_{\mathbb{Q}} ; f\right)^{\sharp}$. Observe that $\pi_{N}\left(X_{\mathbb{Q}}\right)=G_{N}\left(U, X_{\mathbb{Q}} ; f\right)$, where $N=\operatorname{deg} v$.

Remark 3.8. Let $U$ be a finite connected CW complex and $X$ a connected nilpotent CW complex of finite type. Then a $\mathbb{Q}$-localization $h: X \rightarrow X_{\mathbb{Q}}$ induces a map $h_{*}: \mathcal{F}(U, X ; f) \rightarrow \mathcal{F}\left(U, X_{\mathbb{Q}} ; h f\right)$, which is a $\mathbb{Q}$-localization, for any map $f: U \rightarrow X$; see Remark 2.2. Therefore we have

$e v_{*}\left(\pi_{*}(\mathcal{F}(U, X ; f))\right) \otimes \mathbb{Q}=e v_{*} \otimes 1\left(\pi_{*}(\mathcal{F}(U, X ; f)) \otimes \mathbb{Q}\right) \cong e v_{*}\left(\pi_{*}\left(\mathcal{F}\left(U, X_{\mathbb{Q}} ; h f\right)\right)\right)$.

Theorem 3.7 therefore implies the result [35, Theorem 4.1], which was proved by analyzing the construction of the Federer spectral sequence. Smith [36] was also aware of such a generalization.

\section{A detective element and its applications}

Let $f: U \rightarrow X$ be a map from a connected nilpotent space $U$ to a connected rational space $X$. We begin by defining detective elements in the $\psi$-homotopy space $\pi_{\psi}^{*}(X)$ with respect to the triple $(U, X ; f)$. Consider a minimal Sullivan model $i:(\wedge V, d) \longmapsto(\wedge V \otimes \wedge Z, \widehat{d})=(B, \widehat{d})$ for the given map $f: U \rightarrow X$. By definition, the free algebra $(\wedge V \otimes \wedge Z, \widehat{d})$ satisfies the condition that

$$
\widehat{d}(z) \in \wedge^{\geq 1} V \otimes \wedge Z+\wedge V \otimes \wedge^{\geq 2} Z
$$

for any $z \in Z$.

Definition 4.1. An element $v \in \pi_{\psi}^{k}(X)$ for some $k$ is detective with respect to the triple $(U, X ; f)$ if the following conditions $\left(\mathbf{P}_{\mathbf{1}}\right)$ and $\left(\mathbf{P}_{\mathbf{2}}\right)$ hold for an appropriate ordered basis $\left\{u_{i}\right\}_{i \in \mathcal{I}}$ for $V$ such that $\operatorname{deg} u_{i} \leq \operatorname{deg} u_{i+1}$ and $d\left(u_{i+1}\right) \in \wedge V_{i}$ for $i \in \mathcal{I}$.

$\left(\mathbf{P}_{1}\right)$ : We write

$$
d(v)=\sum_{\left(l_{1}, \ldots, l_{r}\right) \in L} q_{l_{1} \cdots l_{r}} u_{l_{1}} \cdots u_{l_{r}}+w
$$


where $L$ is a subset of $\mathcal{I}^{r}, q_{l_{1} \cdots l_{r}}$ are nonzero rational numbers and $w \in \wedge^{\geq r+1} V$. Then there exists an $r$-tuple $\left(i_{1}, \ldots, i_{r}\right) \in L$ such that an element

$$
\alpha_{i_{s}}:=i\left(u_{i_{1}} \cdots u_{i_{s-1}} u_{i_{s+1}} \cdots u_{i_{r}}\right) \in \wedge V \otimes \wedge Z
$$

for some $i_{s}$ does not occur in any image of the differential $\widehat{d}$ as a term. This condition means that $\left\langle\widehat{d h},\left(\alpha_{i_{s}}\right)_{*}\right\rangle=0$ for any $h \in \wedge V \otimes \wedge Z$. Here $\left(\alpha_{i_{s}}\right)_{*}$ denotes the dual element to $\alpha_{i_{s}}$ and $\langle\rangle:, \wedge V \otimes \wedge Z \otimes(\wedge V \otimes \wedge Z)^{\sharp} \rightarrow \mathbb{Q}$ is the pairing.

$\left(\mathbf{P}_{\mathbf{2}}\right)$ : Suppose that $\left(\mathbf{P}_{\mathbf{1}}\right)$ holds. Let $S_{v}$ be the set of basis elements $u_{l_{j}}$ that appear in some term $u_{l_{1}} \cdots u_{l_{r}}$ with $\left(l_{1}, \ldots, l_{r}\right) \in L$ as a factor and let $\left(i_{1}, \ldots, i_{r}\right)$ be the $r$-tuple mentioned in $\left(\mathbf{P}_{\mathbf{1}}\right)$. Then for any $u_{j} \in S_{v}$ and $k$ with $1 \leq k \leq r$ and $k \neq s$,

$$
\widetilde{u}\left(u_{j} \otimes\left(u_{i_{k}}\right)_{*}\right)=\widetilde{u} \gamma \rho\left(u_{j} \otimes\left(u_{i_{k}}\right)_{*}\right)
$$

if $\operatorname{deg} u_{j} \otimes\left(u_{i_{k}}\right)_{*}=0$, where $\widetilde{u}: \wedge\left(V \otimes B_{*}\right) \rightarrow \mathbb{Q}, \rho: \wedge\left(V \otimes B_{*}\right) \rightarrow E$ and $\gamma: E \rightarrow \wedge\left(V \otimes B_{*}\right)$ are DGA maps described in Sections 2 and 3 (see (2.7), (2.8) and $(3.1))$.

Suppose that $\pi_{*}(X)$ is a graded abelian group. We say that an element $x$ in $\pi_{*}(X)$ is detective with respect to $(U, X ; f)$ if the dual element $x^{*}$ in $\pi_{*}(X)^{\sharp} \cong \pi_{\psi}^{*}(X)$ is detective.

Minimal Sullivan models for a given map are unique up to isomorphism; see [9, Theorem 14.12]. This implies that the definition of a detective element does not depend on the choice of the minimal model $i$ for $f$.

Let $x \in \pi_{*}(X)$ be a detective element. We denote by $I(x)$ the subset of integers consisting of the degrees of the $u_{i_{s}}$ in Definition 4.1. By definition, it is evident that $k \leq \operatorname{deg} x$ for any $k \in I(x)$. Moreover, we see that, for any $k \in I(x), k<\operatorname{deg} x$ if $X$ is simply-connected.

The notion of a detective element for a given map $f: U \rightarrow X$ is quite subtle. As is seen below, the algebraic conditions $\left(\mathbf{P}_{\mathbf{1}}\right)$ and $\left(\mathbf{P}_{\mathbf{2}}\right)$ can be derived from suitable geometrical properties of $X, U$ and the map $f$; see Proposition 4.5 and the proof of Theorem 6.1.

The following result yields Theorem 1.1.

Theorem 4.2. Let $f: U \rightarrow X$ be a map from a nilpotent connected space $U$ to a connected rational space $X$ whose fundamental group is abelian. Assume that the condition (2.9) holds and that there exists a detective element $x$ in $\pi_{*}(X)$ with respect to $(U, X ; f)$. Then the evaluation subgroup $G_{k}(U, X ; f)$ is a proper subgroup of $\pi_{k}(X)$ for all $k \in I(x)$.

Before proving Theorem 4.2, we prove a lemma.

Lemma 4.3. Suppose that $v$ is a detective element in $\pi_{\psi}^{*}(X)$ and $\left\{u_{i}\right\}_{i \in \mathcal{I}}$ is a basis for $V$ which satisfies the conditions $\left(\mathbf{P}_{\mathbf{1}}\right)$ and $\left(\mathbf{P}_{\mathbf{2}}\right)$. Let $M_{\widetilde{u}}$ be the ideal of $\wedge\left(V \otimes B_{*}\right)$ generated by the set

$$
\begin{gathered}
\{\omega \mid \operatorname{deg} \omega<0\} \cup\{\delta w \mid \operatorname{deg} \omega=0 \text { or }-1\} \\
\cup\left\{u_{j} \otimes\left(u_{i_{k}}\right)_{*}-\widetilde{u}\left(u_{j} \otimes\left(u_{i_{k}}\right)_{*}\right) \mid u_{j} \in S_{v}, k \neq s, \operatorname{deg} u_{j} \otimes\left(u_{i_{k}}\right)_{*}=0\right\} .
\end{gathered}
$$

Then $M_{\widetilde{u}}$ is closed under differentation and $\rho\left(M_{\widetilde{u}}\right) \subset M_{u}$; see (2.8).

Proof. We first observe that $\delta \widetilde{u}\left(u_{j} \otimes\left(u_{i_{k}}\right)_{*}\right)=\widetilde{u} \delta\left(u_{j} \otimes\left(u_{i_{k}}\right)_{*}\right)=0$ if $\operatorname{deg} u_{j} \otimes\left(u_{i_{k}}\right)_{*}=$ 0 . Thus we have $\delta\left(M_{\widetilde{u}}\right) \subset M_{\widetilde{u}}$. In order to prove Lemma 4.3, it suffices to show 
that, if $\operatorname{deg} u_{j} \otimes\left(u_{i_{k}}\right)_{*}=0$, then

$$
\rho\left(u_{j} \otimes\left(u_{i_{k}}\right)_{*}-\widetilde{u}\left(u_{j} \otimes\left(u_{i_{k}}\right)_{*}\right)\right)=\rho\left(u_{j} \otimes\left(u_{i_{k}}\right)_{*}\right)-u \rho\left(u_{j} \otimes\left(u_{i_{k}}\right)_{*}\right) .
$$

We see that

$$
\begin{aligned}
\rho \widetilde{u}\left(u_{j} \otimes\left(u_{i_{k}}\right)_{*}\right) & =\widetilde{u}\left(u_{j} \otimes\left(u_{i_{k}}\right)_{*}\right) \rho(1) \\
& =\widetilde{u}\left(u_{j} \otimes\left(u_{i_{k}}\right)_{*}\right) \\
& =\widetilde{u} \gamma \rho\left(u_{j} \otimes\left(u_{i_{k}}\right)_{*}\right) .
\end{aligned}
$$

The last equality follows from the condition $\left(\mathbf{P}_{\mathbf{2}}\right)$. Since $u=\widetilde{u} \gamma$ by definition, we have the result.

Proof of Theorem 4.2. We take a minimal Sullivan model $i: A=(\wedge V, d) \longmapsto B=$ $(\wedge V \otimes \wedge Z, \widehat{d})$ for $f$. Consider the DGA $\wedge\left(A \otimes B_{*}\right) / I$ described in Section 3. Let $v$ be the dual element to $x$. By assumption, the conditions $\left(\mathbf{P}_{\mathbf{1}}\right)$ and $\left(\mathbf{P}_{\mathbf{2}}\right)$ hold for $v$. Without loss of generality, we can assume that $\left\langle\widehat{d} \beta,\left(u_{i_{2}} \cdots u_{i_{r}}\right)_{*}\right\rangle=0$ for any $\beta \in \wedge V \otimes \wedge Z$. Thus we see that $\widehat{d}_{*}\left(\left(u_{i_{2}} \cdots u_{i_{r}}\right)_{*}\right)=0$. Lemma 4.3 allows us to obtain the commutative diagram

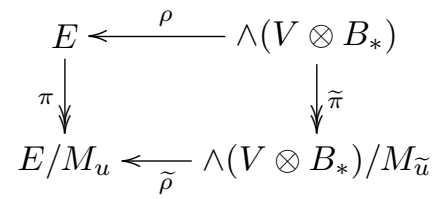

in the category of DGA's, where $\tilde{\pi}$ is the natural projection and $\widetilde{\rho}$ is a DGA map induced by $\rho$.

Choose the element of the form $v \otimes\left(u_{i_{2}} \cdots u_{i_{r}}\right)_{*}$ in $\wedge\left(A \otimes B_{*}\right) / I \cong \wedge\left(V \otimes B_{*}\right)$. From (2.1), we see that

$$
\begin{aligned}
\delta \pi \rho\left(v \otimes\left(u_{i_{2}} \cdots u_{i_{r}}\right)_{*}\right) & =\pi \rho \delta\left(v \otimes\left(u_{i_{2}} \cdots u_{i_{r}}\right)_{*}\right) \\
& =\tilde{\rho} \tilde{\pi}\left(\sum_{\left(l_{1}, \ldots, l_{r}\right) \in L} q_{l_{1} \cdots l_{r}} u_{l_{1}} \cdots u_{l_{r}} \otimes\left(u_{i_{2}} \cdots u_{i_{r}}\right)_{*}\right. \\
& \left.+w \otimes\left(u_{i_{2}} \cdots u_{i_{r}}\right)_{*}\right) \\
& =q_{i_{1} \cdots i_{r}}^{\prime}\left(u_{i_{1}} \otimes 1_{*}\right)+\sum_{j_{1}} q_{j_{1} i_{2} \cdots i_{r}}^{\prime}\left(u_{j_{1}} \otimes 1_{*}\right)+\gamma
\end{aligned}
$$

where $\gamma$ is a decomposable element, $u_{i_{1}} \neq u_{j_{1}}$ for any $j_{1}, q_{i_{1} \cdots i_{r}}^{\prime}=m q_{i_{1} \cdots i_{r}}$ and $q_{j_{1} i_{2} \cdots i_{r}}^{\prime}=n q_{j_{1} i_{2} \cdots i_{r}}$ for appropriate nonzero rational numbers $m$ and $n$. It follows from Lemma 3.5 that $Q(\widetilde{e v})\left(q_{i_{1} \cdots i_{r}}^{\prime} u_{i_{1}}+\sum_{j_{1}} q_{j_{1} i_{2} \cdots i_{r}}^{\prime} u_{j_{1}}\right)=0$. We have the result.

We here give a sufficient condition for $\left(\mathbf{P}_{\mathbf{2}}\right)$.

Lemma 4.4. Let $(\wedge V, d)$ be a minimal model for $X$ and $\left\{v_{i}\right\}_{i \in \mathcal{I}}$ an ordered basis for $V$ such that $\operatorname{deg} v_{i} \leq \operatorname{deg} v_{i+1}$ and $d\left(v_{i+1}\right) \in \wedge V_{i}$. Suppose that the induced map $\pi_{\psi}^{*}(f): \pi_{\psi}^{i}(X) \rightarrow \pi_{\psi}^{i}(\bar{U})$ between the $\psi$-homotopy spaces is a monomorphism for $i$ less than or equal to some integer $k$. Then for any element $v_{i} \otimes e \in V \otimes B_{*}$ with $\operatorname{deg} v_{i} \otimes e=0$,

$$
\widetilde{u}\left(v_{i} \otimes e\right)=\widetilde{u} \gamma \rho\left(v_{i} \otimes e\right),
$$

if $\operatorname{deg} v_{i} \leq k$. 
Proposition 4.5. Let $v$ be an element of $\pi_{\psi}^{*}(X)$ which satisfies the condition $\left(\mathbf{P}_{\mathbf{1}}\right)$. If the induced map $\pi_{\psi}^{*}(f): \pi_{\psi}^{i}(X) \rightarrow \pi_{\psi}^{i}(U)$ is a monomorphism for $i \leq \operatorname{deg} v$, then the condition $\left(\mathbf{P}_{\mathbf{2}}\right)$ holds.

Proof. Under the notation of the condition $\left(\mathbf{P}_{\mathbf{2}}\right)$, we see that $\operatorname{deg} u_{j} \leq \operatorname{deg} x$. The result follows from Lemma 4.4 .

Proof of Lemma 4.4. We write $B=C \oplus \widehat{d} C \oplus H$ for which $\widehat{d}: C \rightarrow \widehat{d} C$ is an isomorphism and $\widehat{d} H=0$. Let $\left\{b_{k}^{\prime}, \widehat{d b}_{k}^{\prime}, c_{j}^{\prime}\right\}_{k, j}$ be a basis for $B$ which satisfies the condition that $b_{k}^{\prime} \in C$ and $c_{j}^{\prime} \in H$. Let $\left\{b_{k}\right\}_{k},\left\{a_{k}\right\}_{k}$ and $\left\{c_{j}\right\}_{j}$ be dual bases of $\left\{b_{k}^{\prime}\right\}_{k},\left\{\widehat{d b}_{k}^{\prime}\right\}_{k}$ and $\left\{c_{j}^{\prime}\right\}_{j}$, respectively. Thus $\left\{b_{k}, a_{k}, c_{j}\right\}_{k, j}$ is a basis for $B_{*}=C_{*} \oplus(\widehat{d} C)_{*} \oplus H_{*}=\widehat{d}_{*}(\widehat{d} C)_{*} \oplus(\widehat{d} C)_{*} \oplus H_{*}$. Observe that $\widehat{d}_{*} a_{k}=b_{k}$.

We will prove Lemma 4.4 by induction on $i \in \mathcal{I}$. Suppose that $\widetilde{u}\left(v_{l} \otimes e\right)=$ $\tilde{u} \gamma \rho\left(v_{l} \otimes e\right)$ for any element $v_{l} \otimes e \in V \otimes B_{*}$ with $\operatorname{deg} v_{l} \otimes e=0$ and $l<i$. We prove now that $\widetilde{u}\left(v_{i} \otimes e\right)=\widetilde{u} \gamma \rho\left(v_{i} \otimes e\right)$ when $\operatorname{deg} v_{i} \otimes e=0$, first for $e=b_{k}$, then for $e=a_{k}$ and finally for $e=c_{j}$.

Consider an element of the form $v_{i} \otimes b_{k}$ of degree zero. Since $(-1)^{\left|v_{i}\right|} v_{i} \otimes b_{k}=$ $\delta\left(v_{i} \otimes a_{k}\right)-d v_{i} \otimes a_{k}$, it follows that

$$
(-1)^{\left|v_{i}\right|} \widetilde{u}\left(v_{i} \otimes b_{k}\right)=\delta \widetilde{u}\left(v_{i} \otimes a_{k}\right)-\widetilde{u}\left(d v_{i} \otimes a_{k}\right)=-\widetilde{u}\left(d v_{i} \otimes a_{k}\right) .
$$

On the other hand,

$$
(-1)^{\left|v_{i}\right|} \widetilde{u} \gamma \rho\left(v_{i} \otimes b_{k}\right)=\widetilde{u} \gamma \rho\left(\delta\left(v_{i} \otimes a_{k}\right)\right)-\widetilde{u} \gamma \rho\left(d v_{i} \otimes a_{k}\right)=-\widetilde{u}\left(d v_{i} \otimes a_{k}\right)
$$

by the induction hypothesis. This yields that $\widetilde{u}\left(v_{i} \otimes b_{k}\right)=\widetilde{u} \gamma \rho\left(v_{i} \otimes b_{k}\right)$.

We write $\widehat{d} b_{k}^{\prime}=c v_{i}+q$, where $v_{i}$ and $q$ are linearly independent. Thus $a_{k}=$ $\left(d b_{k}^{\prime}\right)_{*}=c\left(v_{i}\right)_{*}+q_{*}$. Assume that $\widetilde{u}\left(v_{i} \otimes a_{k}\right) \neq 0$. The definition of $\widetilde{u}$ implies that $0 \neq\left\langle v_{i}, a_{k}\right\rangle=\left\langle v_{i}, c\left(v_{i}\right)_{*}+q_{*}\right\rangle=c$. Moreover $\widehat{d}_{0}\left(b_{k}^{\prime}\right) \in V$. These facts enable us to deduce that $\pi_{\psi}^{*}(f): \pi_{\psi}^{\left|v_{i}\right|}(X) \rightarrow \pi_{\psi}^{\left|v_{i}\right|}(U)$ is not a monomorphism, which is a contradiction. We conclude that $\widetilde{u}\left(v_{i} \otimes a_{k}\right)=0=\widetilde{u} \gamma \rho\left(v_{i} \otimes a_{k}\right)$.

Consider an element $v_{i} \otimes c_{j}$ with $\operatorname{deg} v_{i} \otimes c_{j}=0$. Using the generator $w_{i j}$ of $\wedge\left(V \otimes B_{*}\right)$ described in Section 2, we write $v_{i} \otimes c_{j}=w_{i j}-x_{i j}$, where $x_{i j}$ is an element in $\wedge\left(V_{i-1} \otimes B_{*}\right)$. Since $\gamma \rho\left(w_{i j}\right)=w_{i j}$, it follows that

$$
\begin{aligned}
\widetilde{u}\left(v_{i} \otimes c_{j}\right) & =\widetilde{u}\left(w_{i j}\right)-\widetilde{u}\left(x_{i j}\right) \\
& =\widetilde{u} \gamma \rho\left(w_{i j}\right)-\widetilde{u} \gamma \rho\left(x_{i j}\right) \\
& =\widetilde{u} \gamma \rho\left(v_{i} \otimes c_{j}\right) .
\end{aligned}
$$

It turns out that $\widetilde{u}\left(v_{i} \otimes e\right)=\widetilde{u} \gamma \rho\left(v_{i} \otimes e\right)$ for any element $v_{i} \otimes e \in V \otimes B_{*}$ with degree zero.

The first step of the induction is obtained by the same argument as above; that is, $\widetilde{u}\left(v_{1} \otimes e\right)=\widetilde{u} \gamma \rho\left(v_{1} \otimes e\right)$ if $\operatorname{deg} v_{1} \otimes e=0$. This completes the proof.

Example 4.6. Let $S U(3) \rightarrow P \stackrel{p}{\rightarrow} S^{3} \times S^{3}$ be the principal bundle with the classifying map $S^{3} \times S^{3} \stackrel{\pi}{\rightarrow} S^{3} \times S^{3} / S^{3} \vee S^{3} \cong S^{6} \stackrel{j}{\rightarrow} B S U(3)$, where $\pi$ is the projection and $j$ is a generator of $\pi_{6}(B S U(3)) \otimes \mathbb{Q}$. Then the bundle has a model of the form

$$
\left(\wedge\left(x_{3}, x_{5}\right), 0\right) \ll\left(\wedge(u, v) \otimes \wedge\left(x_{3}, x_{5}\right), d\right) \prec<(\wedge(u, v), 0)
$$

in which $\operatorname{deg} x_{i}=i, \operatorname{deg} u=\operatorname{deg} v=3, d(u)=d(v)=d\left(x_{3}\right)=0$ and $d\left(x_{5}\right)=u v$; see $\left[9\right.$, Example 4, page 220]. Let $q: S^{3} \times S^{3} \rightarrow S^{3}$ denote the projection on the 
second factor. We obtain the bundle

$$
S U(3) \times S^{3} \stackrel{i}{\rightarrow} P \stackrel{q p}{\rightarrow} S^{3}
$$

which admits a model $\left(\wedge\left(x_{3}, x_{5}, u\right), 0\right) \ll \pi\left(\wedge(u, v) \otimes \wedge\left(x_{3}, x_{5}\right), d\right) \ll<(\wedge(v), 0)$ with $d\left(x_{5}\right)=u v$. Observe that the projection $\pi$ is a Sullivan representative for the inclusion $i$. The map $\pi$ has a Sullivan model of the form

$$
j:\left(\wedge(u, v) \otimes \wedge\left(x_{3}, x_{5}\right), d\right)>\longrightarrow\left(\wedge(u, v) \otimes \wedge\left(x_{3}, x_{5}\right) \otimes \wedge(w), \widehat{d}\right)=:(A, \widehat{d}),
$$

in which the differential $\widehat{d}$ is defined by $\widehat{d}(w)=v$. To see this, we define a morphism of DGA's $\varphi:(A, \widehat{d}) \rightarrow\left(\wedge\left(x_{3}, x_{5}, u\right), 0\right)$ by $\varphi(w)=\varphi(v)=0, \varphi(u)=u$ and $\varphi\left(x_{i}\right)=$ $x_{i}$ for $i=3,5$. It is readily seen that $\varphi$ is a quasi-isomorphism with $\pi=\varphi \circ j$. This implies that $j$ is a Sullivan model for the map $i$.

By using the Sullivan model $j$, we see that the dual element $x_{5 *} \in \pi_{5}\left(P_{\mathbb{Q}}\right)$ is detective with respect to the triple $\left(\left(S U(3) \times S^{3}\right), P_{\mathbb{Q}} ; e_{P} \circ i\right)$. In fact, $\widehat{d}\left(x_{5}\right)=u v$ and the element $u$ does not occur in any image of $\widehat{d}$ as a term. Thus the condition $\left(\mathbf{P}_{\mathbf{1}}\right)$ holds. Moreover we can choose the set of generators $\left\{w_{i j}\right\}$ of $E$ as in Section 2 extending linearly independent elements $v \otimes u_{*}, u \otimes u_{*}$ and $w \otimes u_{*}$. Therefore $\widetilde{u}\left(z \otimes u_{*}\right)=\widetilde{u} \gamma \rho\left(z \otimes u_{*}\right)$ for $z=v, u$ and $w$ and hence the condition $\left(\mathbf{P}_{\mathbf{2}}\right)$ holds.

Theorem 1.1 yields that $G_{*}\left(\left(S U(3) \times S^{3}\right), P_{\mathbb{Q}} ; e_{P} \circ i\right)$ is a proper subgroup of $\pi_{*}\left(P_{\mathbb{Q}}\right)$ and hence the map $i$ is not cyclic by Corollary 1.4. It turns out that there is no action on $P$ of the group $S U(3) \times S^{3}$ for which $i$ is an orbit map.

Remark 4.7. In Example 4.6 since $\widehat{d}(w)=v$, it follows that $\pi_{\psi}^{*}(j): \pi_{\psi}^{3}(P) \rightarrow$ $\pi_{\psi}^{3}\left(S U(3) \times S^{3}\right)$ is not a monomorphism. Thus Proposition 4.5 is not applicable to show that $x$ is detective because $\operatorname{deg} x_{5}=5$.

We close this section with another application of a detective element which is related to characterization of Hopf spaces.

We see that $G_{n}(X) \subset G_{n}(U, X ; f) \subset \pi_{n}(X)$ for any map $f: U \rightarrow X$ by [14, Proposition 1.2]. Moreover, $G_{*}(X)=\pi_{*}(X)$ if $X$ is a Hopf space by [14, Proposition 2.2]. It follows that $G_{*}(U, X ; f)=\pi_{*}(X)$ for any map $f: U \rightarrow X$ if $X$ is a Hopf space.

The converse holds in some special cases. For example, it is shown by Haslam in [18] that if $X$ is a simply-connected finite CW complex with $G_{*}\left(X_{\mathbb{Q}}\right)=\pi_{*}\left(X_{\mathbb{Q}}\right)$, then $X_{\mathbb{Q}}$ is a Hopf space. We describe a necessary and sufficient condition for a rational space to be a Hopf space in terms of an evaluation subgroup. To this end, we need a definition.

A map $f: U \rightarrow X$ is said to have a rational section if a minimal Sullivan model $m: \wedge V \rightarrow \wedge V \otimes \wedge Z$ for $f$ admits a left inverse; that is, there exists a DGA map $p: \wedge V \otimes \wedge Z \rightarrow \wedge V$ such that $p \circ m=1_{\wedge V}$. Suppose that $U$ is connected and $X$ is simply-connected. Then we observe that $f: U \rightarrow X$ has a rational section if the map $f$ has a right homotopy inverse $g: X \rightarrow U$.

Theorem 4.8. Let $U, X$ and $f: U \rightarrow X$ be as in Theorem 4.2. Suppose further that $f: U \rightarrow X$ has a rational section. Then $X$ is a Hopf space if and only if $G_{*}(U, X ; f)=\pi_{*}(X)$.

Proof. It suffices to prove the "if" part. Let $m:(\wedge V, d) \longmapsto(\wedge V \otimes \wedge Z, \widehat{d})$ be a minimal Sullivan model for $f$. By assumption, the map $m$ admits a left inverse 
$p:(\wedge V \otimes \wedge Z, \widehat{d}) \rightarrow(\wedge V, d)$. Let $\left\{\widetilde{z}_{j}\right\}_{j \in J}$ be a basis for $Z$. We can regard the set $\left\{z_{j}\right\}_{j \in J}$ consisting of elements $z_{j}=\widetilde{z}_{j}-m p \widetilde{z}_{j}$ as another basis of $Z$. It is immediate that $p z_{j}=0$.

Assume that $X$ is not a Hopf space, so that the differential $d$ of the model $(\wedge V, d)$ is not strictly zero. We shall prove that the lowest-degree non-cycle $v$ in $V$ is detective.

Let $\left\{u_{i}\right\}$ be an ordered basis of $V$ such that $\operatorname{deg} u_{i} \leq \operatorname{deg} u_{i+1}$ and $d\left(u_{i+1}\right) \in \wedge V_{i}$. Write $d v=\sum q_{i_{1} \ldots i_{r}} u_{i_{1}} \cdots u_{i_{r}}+w$, where $w \in \wedge^{\geq r+1} V$ and the $q_{i_{1} \ldots i_{r}}$ are nonzero rational numbers. Letting $\nu$ be the element $m\left(u_{i_{2}} \cdots u_{i_{r}}\right)$, suppose that $\left\langle\widehat{d h}, \nu_{*}\right\rangle \neq 0$ for some $h \in \wedge V \otimes \wedge Z$. If $h$ is in the image of $m$, then $\widehat{d h}=0$ since $\operatorname{deg} h<\operatorname{deg} v$. Thus it follows that $h$ is in the ideal $J$ of $\wedge V \otimes \wedge Z$ generated by $\left\{z_{j}\right\}_{j \in J}$. We write

$$
\widehat{d h}=s \nu+\sum \eta_{k}+\Phi
$$

where $s$ is a nonzero rational number, $\eta_{k} \in \wedge V$ and $\Phi \in J$. We assume further that $\nu$ and $\eta_{k}$ 's are linearly independent. Then it is readily seen that $p \widehat{d h}=d p h=0$. Therefore, we have $0=p\left(s \nu+\sum \eta_{k}+\Phi\right)=s \nu+\sum \eta_{k}$, which is a contradiction. We can conclude that $\left\langle\widehat{d} h, \nu_{*}\right\rangle=0$. It follows from Proposition 4.5 that the condition $\left(\mathbf{P}_{\mathbf{2}}\right)$ holds. This implies that the dual element of $v$ is detective with respect to $(U, X ; f)$. By virtue of Theorem 1.1, we have the result.

Remark 4.9. Suppose that a based map $f: U \rightarrow X$ has a right homotopy inverse in the category of based spaces. Then we see that $G_{*}(U, X ; f)=G_{*}(X)$. In fact, if $g$ : $X \rightarrow U$ is a right homotopy inverse of $f$, then the induced map $f^{*}: \mathcal{F}\left(X, X ; i d_{X}\right) \rightarrow$ $\mathcal{F}(U, X ; f)$ has the left homotopy inverse $g^{*}: \mathcal{F}(U, X ; f) \rightarrow \mathcal{F}\left(X, X ; i d_{X}\right)$; that is, $g^{*} \circ f^{*}$ is homotopic to the identity map on $\mathcal{F}(X, X)$ the function space of all continuous maps from $X$ to itself. Thus we have the following digram.

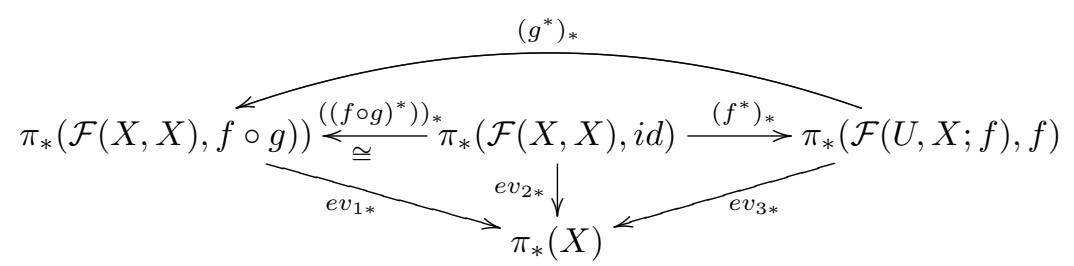

in which three inner triangles and the outer triangle are commutative. Here each $e v_{i}$ denotes the evaluation map. The commutativity of the diagram enables us to conclude that $G_{*}(X)=\operatorname{Im} e v_{2 *}=\operatorname{Im} e v_{3 *}=G_{*}(U, X ; f)$. If moreover $X$ is a finite simpy-connected CW complex, then Theorem 4.8 follows from Haslam's result. We stress that such a finiteness condition on $X$, the simply-conectedness and the existence of a geometrical section of the map $f$ are not required in Theorem 4.8 .

Recall that $X$ is a $G$-space if $G_{n}(X)=\pi_{n}(X)$ for any $n \geq 1$. In [33], Siegel has constructed a non simply-connected space which is a $G$-space but not a Hopf space. By generalizing the construction, we here give a non simply-connected homogeneous space $M$ which is a rational Hopf space; that is, the localization $M_{\mathbb{Q}}$ is a Hopf space, but not a Hopf space itself.

Let $G$ be a compact simply-connected Lie group and $T$ an $l$-dimensional torus subgroup of $G$. Let $\iota: T \rightarrow G$ be the inclusion. Define a embedding $j: T \rightarrow G \times T$ 
by

$$
j\left(e^{i \theta_{1}}, \ldots, e^{i \theta_{l}}\right)=\left(\iota\left(\left(e^{i m_{1} \theta_{1}}, \ldots, e^{i m_{l} \theta_{l}}\right)\right), e^{i n_{1} \theta_{1}}, \ldots, e^{i n_{l} \theta_{l}}\right),
$$

where $m_{1}, \ldots, m_{l}, n_{1}, \ldots, n_{l}$ are non-zero integers and $m_{k}$ is prime to $n_{k}$ for any $1 \leq k \leq l$.

By applying Theorem 4.8, we have the following proposition.

Proposition 4.10. The homogeneous space $M:=G \times T / j(T)$ is a non simplyconnected $G$-space and a rational Hopf space.

Proof. Since the induced map $j_{*}: \pi_{n}(T) \rightarrow \pi_{n}(G \times T)$ is injective for all $n \geq 1$, it follows from [33, Theorem 2.3] that $M$ is a $G$-space. Observe that $\pi_{1}(M)$ is a nontrivial abelian group and that the localization $e: M \rightarrow M_{\mathbb{Q}}$ induces an monomorphism $e_{*}: G_{n}(M) \otimes \mathbb{Q} \rightarrow G_{n}\left(M_{\mathbb{Q}}\right)$; see [30, Theorem 4.1]. We see that $G_{n}\left(M_{\mathbb{Q}}\right)=\pi_{n}\left(M_{\mathbb{Q}}\right)$ for any $n$ because $G_{n}(M)=\pi_{n}(M)$ for any $n$. Theorem 4.8 yields that $M_{\mathbb{Q}}$ is a Hopf space.

Proposition 4.11. Suppose that, for a prime number $p$, the integral homology of $G$ is $p$-torsion free and $\frac{\operatorname{dim} G}{2}+1<p$. Assume further that at least one of the integers $n_{1}, \ldots, n_{l}$ is divisible by $p$. Then $M$ is not a Hopf space.

Proof. Consider the fibration of the form $M \rightarrow B T \stackrel{B j}{\rightarrow} B(G \times T)$. This gives rise to the Eilenberg-Moore spectral sequence $\left\{E_{r}, d_{r}\right\}$ converging to $H^{*}(M ; \mathbb{Z} / p)$ as an algebra with

$$
E_{2}^{*, *} \cong \operatorname{Tor}_{H^{*}(B G ; \mathbb{Z} / p) \otimes H^{*}(B T ; \mathbb{Z} / p)}^{* *}\left(H^{*}(B T ; \mathbb{Z} / p), \mathbb{Z} / p\right)
$$

as a bigraded algebra. Observe that the $H^{*}(B G ; \mathbb{Z} / p) \otimes H^{*}(B T ; \mathbb{Z} / p)$-action on $H^{*}(B T ; \mathbb{Z} / p)$ is given by the composite map

$$
\begin{aligned}
H^{*}(B G ; \mathbb{Z} / p) \otimes H^{*}(B T ; \mathbb{Z} / p) \otimes H^{*}(B T ; \mathbb{Z} / p) & \\
& \downarrow(B j)^{*} \otimes 1 \\
H^{*}(B T ; \mathbb{Z} / p) \otimes H^{*}(B T ; \mathbb{Z} / p) \longrightarrow{ }^{\circ} &
\end{aligned}
$$

where $c$ denotes the cup product of $H^{*}(B T ; \mathbb{Z} / p)$. Since $H_{*}(G)$ is $p$-torsion free, the cohomology $H^{*}(B G ; \mathbb{Z} / p)$ is isomorphic to a polynomial algebra, say, $\mathbb{Z} / p\left[x_{1}, \ldots, x_{n}\right]$. We can write $H^{*}(B T ; \mathbb{Z} / p) \cong \mathbb{Z} / p\left[t_{1}, \ldots, t_{l}\right]$ for which $\operatorname{deg} t_{k}=2$ for any $1 \leq k \leq l$. It follows from the definition of the embedding $j$ that $(B j)^{*}\left(t_{k}\right)=n_{k} t_{k}$ for $1 \leq k \leq l$. Without loss of generality, we assume that $n_{1}$ is divisible by $p$. This implies that $t_{1}$ is one of the generators of the algebra $E_{2}^{0, *}$ in $E_{2}^{0,2}$. The result $[28,8.2$. Theorem] enables us to conclude that the spectral sequence $\left\{E_{r}, d_{r}\right\}$ collapses at the $E_{2}$-term. The edge homomorphism

$$
E_{2}^{0, *} \rightarrow E_{\infty}^{0, *} \cong E_{0}^{0, *} \subset H^{*}(M ; \mathbb{Z} / p)
$$

is a morphism of algebras in general; see [34, Proposition 4.2]. Hence $E_{2}^{0, *}$ is regarded as a subalgebra of $H^{*}(M ; \mathbb{Z} / p)$. Since $M$ is a manifold of dimension $\operatorname{dim} G$, we can choose the least integer $s$ such that $t_{1}^{s}=0$ in $H^{*}(M ; \mathbb{Z} / p)$. It follows that $2(s-1) \leq \operatorname{dim} G$ and hence $s \leq \frac{\operatorname{dim} G}{2}+1<p$ by assumption.

Suppose that $M$ is a Hopf space with a product $h: M \times M \rightarrow M$. We have

$$
0=h^{*}\left(t_{1}^{s}\right)=h^{*}\left(t_{1}\right)^{s}=\left(t_{1} \otimes 1+1 \otimes t_{1}\right)^{s}=\cdots+{ }_{s} \mathrm{C}_{k} t_{1}^{s-k} \otimes t_{1}^{k}+\cdots \neq 0,
$$

which is a contradiction. 
Remark 4.12. The homogeneous space of the form $S O(3) \times S^{1} / j\left(S^{1}\right)$ constructed with an appropriate embedding $j: S^{1} \rightarrow S O(3) \times S^{1}$ in [33, 2.4 Example] satisfies the conditions in Proposition 4.11 for $p=3$. In fact $H_{*}(S O(3))$ is 3 -torsion free and $\operatorname{dim} S O(3)=3$.

\section{Non Gottlieb Elements Detected By Whitehead PRoducts}

The following result is the key to proving Theorem 1.2.

Theorem 5.1. Let $f: U \rightarrow X$ be a map between connected spaces $U$ and $X$ which are not necessarily rational. If for elements $g_{1}, \ldots, g_{n-1}$ in $\pi_{*}(U)$, the $(n-1)$ th order Whitehead product $\left[g_{1}, \ldots, g_{n-1}\right]$ contains zero, then so does $\left[f_{*}\left(g_{1}\right), \ldots, f_{*}\left(g_{n-1}\right), x\right]$ for any $x \in G_{*}(U, X ; f)$.

Remark 5.2. With the same notation as above, suppose that $\left[g_{i_{1}}, g_{i_{2}}, \ldots, g_{i_{k}}\right]=\{0\}$ for any subset $\left\{g_{i_{1}}, \ldots, g_{i_{k}}\right\}$ of $\left\{g_{1}, \ldots, g_{n-1}\right\}$ with $i_{1}<i_{2}<\cdots<i_{k}$ and $k<n-1$. Then $\left[g_{1}, \ldots, g_{n-1}\right]$ is non-empty. This follows from [32, Theorem (2.7)]; see also the comment after $[32$, Theorem (2.7)].

Proof of Theorem 5.1. The usual argument on composing a Gottlieb element with an element in the homotopy group is applicable to our case; see, for example, [13] [9, Proposition 28.7]. We put $k_{i}=\operatorname{deg} g_{i}$ and $k_{n}=\operatorname{deg} x$. Let $T_{m}$ denote the fat wedge, which is a subspace of $P_{m}=S^{k_{1}} \times \cdots \times S^{k_{m}}$. Since $\left[g_{1}, \ldots, g_{n-1}\right]$ is non-empty and contains zero, it follows from [32, Theorem (2.4)] that the map $g_{1} \vee \cdots \vee g_{n-1}$ extends to a map $\varphi: P_{n-1} \rightarrow U$. The element $f \vee x: U \vee S^{k_{n}} \rightarrow X$ has an extension $\psi: U \times S^{k_{n}} \rightarrow X$ because $x \in G_{*}(U, X ; f)$. Therefore we see that the composition $\psi \circ(\varphi \times 1): P_{n} \rightarrow X$ is an extension of the map $(f \vee x) \circ\left(g_{1} \vee \cdots \vee g_{n-1} \vee 1\right)=$ $f_{*}\left(g_{1}\right) \vee \cdots \vee f_{*}\left(g_{n-1}\right) \vee x$.

Let $\sigma$ be a permutation of the set $\{1, \ldots, r\}$ of $r$ integers. We define a map $\sigma_{*}: \times_{i=1}^{r} S^{n_{i}} \rightarrow \times_{i=1}^{r} S^{n_{\sigma(i)}}$ by permuting the coordinate by $\sigma$. For the generators $\mu \in H_{*}\left(\times_{i=1}^{r} S^{n_{i}}\right)$ and $\mu^{\prime} \in H_{*}\left(\times_{i=1}^{r} S^{n_{\sigma(i)}}\right)$ mentioned in Introduction, we see that $(-1)^{\varepsilon(\sigma)} \mu^{\prime}=\sigma_{*}(\mu)$. Here $\varepsilon(\sigma)$ is the integer defined by the formula $u_{1} \cdots u_{r}=(-1)^{\varepsilon(\sigma)} u_{\sigma(1)} \cdots u_{\sigma(r)}$ in the graded commutative free algebra generated by elements $u_{1}, \ldots, u_{r}$ with $\operatorname{deg} u_{i}=\operatorname{deg} x_{i}$. The definition of the higher order Whitehead product enables us to conclude that, if $x \in\left[x_{1}, \ldots, x_{r}\right]$, then $(-1)^{\varepsilon(\sigma)} x \in\left[x_{\sigma(1)}, \ldots, x_{\sigma(r)}\right]$. Thus in order to prove Theorem 1.2, it suffices to show that $\left[x_{1}, \ldots, x_{r-1}, x_{r}\right]=\{0\}$ for any $x_{1}, \ldots, x_{r-1}$ in $\pi_{*}(X)$ if $x_{r} \in G_{*}(U, X ; f)$ under the assumption in the theorem.

Proof of Theorem 1.2. We first consider the Whitehead products in $X$. It follows from the assumption on the Whitehead products in $U$ and [32, Theorem $(2.1)(\mathrm{d})]$ that all Whitehead products of order $<r$ in $X$ contain zero. By applying [1, Corollary 6.5] repeatedly, we see that all of those actually vanish; see Remark 1.3.

Let $x_{1}, \ldots, x_{r}$ be elements in $\pi_{*}(X)$. Suppose that $x_{r} \in G_{*}(U, X ; f)$. By assumption, we can choose elements $g_{i} \in \pi_{*}(U)(1 \leq i \leq r-1)$ so that $f_{*}\left(g_{i}\right)=x_{i}$. Theorem 5.1 allows us to conclude that $\left[x_{1}, \ldots, x_{r}\right]$ contains zero since $\left[g_{1}, \ldots, g_{r-1}\right]=\{0\}$. By applying $\left[1\right.$, Corollary 6.5] again, we see that $\left[x_{1}, \ldots, x_{r}\right]=\{0\}$. This completes the proof.

By using Theorem 5.1 in the case $n=2$, we can recover the result [35, Theorem 4.2] concerning the vanishing of an evaluation subgroup. The proof of the following proposition is left to the reader. 
Proposition 5.3. (cf. [35, Theorem 4.2]) Let $U$ and $X$ be connected based spaces and $f: U \rightarrow X$ a based map. If there exist nonzero elements $x_{i}$ in $\pi_{*}(U)$ such that $\cap_{i}\left(\operatorname{Ker} \operatorname{ad} f_{*}\left(x_{i}\right)\right)=0$, then $G_{*}(U, X ; f)=0$. Here $\operatorname{ad} f_{*}\left(x_{i}\right): \pi_{*}(X) \rightarrow \pi_{*}(X)$ is the homomorphism defined by ad $f_{*}\left(x_{i}\right)(z)=\left[f_{*}\left(x_{i}\right), z\right]$.

We end this section with some results on rational higher order Whitehead products.

Proposition 5.4. Assume that $n \geq 3, m \geq 2$ and $m_{i} \geq 2$ for all $1 \leq i \leq n$. Let $x_{i}$ be an element of $\pi_{m_{i}}\left(S_{\mathbb{Q}}^{m}\right)$ for $i=1,2, \ldots, n$. Then

(1) If $m$ is odd, then $\left[x_{1}, \ldots, x_{n}\right]$ is non-empty and $\left[x_{1}, \ldots, x_{n}\right]=\{0\}$.

(2) If $m$ is even and $\left[x_{1}, \ldots, x_{n}\right]$ is non-empty, then $\left[x_{1}, \ldots, x_{n}\right]$ contains zero.

Proof. If $m$ is odd, then $S_{\mathbb{Q}}^{m}$ is a Hopf space. Hence we have the result (1) by [32, Theorem (2.4)]. Suppose that $m$ is even. For dimensional reasons, it suffices to consider the case where $\operatorname{deg} x_{1}=m$ and $\operatorname{deg} x_{1}+\operatorname{deg} x_{2}+\cdots+\operatorname{deg} x_{n}-1=2 m-1$. It is readily seen that each $x_{j}=0$ for $j>1$. Since $0 \in G_{i}\left(S_{\mathbb{Q}}^{m}\right)$ for any $i$, by applying Theorem 5.1 repeatedly to the case where $f=i d: S_{\mathbb{Q}}^{m} \rightarrow S_{\mathbb{Q}}^{m}$, we have $\left[x_{1}, \ldots, x_{n}\right] \ni 0$.

Corollary 5.5. Let $X$ be a rational space. If $n \geq 3$ and $m$ is odd, then for any element $x \in \pi_{m}(X)$, the nth order Whitehead product $[x, x, \ldots, x]$ is well-defined and contains zero.

Proof. Let $j_{m}: S^{m} \rightarrow S_{\mathbb{Q}}^{m}$ be the rationalization map. We see that $x=x_{\mathbb{Q}} \circ j_{m}$, where $x_{\mathbb{Q}}$ denotes the $\mathbb{Q}$-localization of $x$. Proposition 5.4 and [32, Theorem (2.1) (d)] imply that

$$
\{0\}=x_{\mathbb{Q}} \circ\left[j_{m}, j_{m}, \cdots, j_{m}\right] \subset\left[x_{\mathbb{Q}} \circ j_{m}, x_{\mathbb{Q}} \circ j_{m}, \cdots, x_{\mathbb{Q}} \circ j_{m}\right]=[x, x, \cdots, x] .
$$

Corollary 5.6. Assume that $n \geq 3$ and $m_{i} \geq m \geq 2$. If $x_{i}: S^{m_{i}} \rightarrow S^{m}(i=$ $1,2, \cdots, n)$, then $\left[x_{1}, \cdots, x_{n}\right]$ is of finite order if it is well-defined.

Proof. Let $j_{m}: S^{m} \rightarrow S_{\mathbb{Q}}^{m}$ be the rationalization map. For dimensional reasons, it follows from Proposition 5.4 that $j_{m} \circ\left[x_{1}, \cdots, x_{n}\right] \subset\left[j_{m} \circ x_{1}, \cdots, j_{m} \circ x_{n}\right]=\{0\}$.

\section{A GEOMETRIC INTERPRETATION OF A DETECTIVE ELEMENT}

In this section, we prove the following theorem.

Theorem 6.1. Let $U$ and $X$ be simply-connected rational spaces and let $f$ be as in Theorem 1.2. Assume that all Whitehead products of order less than $r$ vanish in $\pi_{*}(U)$. Then a nonzero element $x$ in $\left[x_{1}, \ldots, x_{r}\right]$ for some elements $x_{i}$, if any, is detective with respect to $(U, X ; f)$. Moreover, $\operatorname{deg} x_{i} \in I(x)$ for any $i$.

We begin by recalling the result in [1] concerning rational higher order Whitehead products. Fix positive integers $n_{1}, \ldots, n_{r}$. Then we define a function $K$ from the set $M(r, \mathbb{Q})$ of $r \times r$-matrices to $\mathbb{Q}$ by

$$
K\left(\left(a_{i j}\right)\right)=\sum_{\sigma \in \Sigma_{r}}(-1)^{\varepsilon(\sigma)} a_{1 \sigma(1)} \cdots a_{r \sigma(r)} .
$$

Here $\Sigma_{r}$ denotes the symmetric group and the integer $\varepsilon(\sigma)$ is characterized by the formula $u_{1} \cdots u_{r}=(-1)^{\varepsilon(\sigma)} u_{\sigma(1)} \cdots u_{\sigma(r)}$ for elements $u_{1}, \ldots, u_{r}$ with $\operatorname{deg} u_{i}=n_{i}$ in a graded commutative algebra. 
Let $(\wedge V, d)$ be the minimal model for a simply-connected space $X$. We fix a basis $\left\{u_{i}\right\}$ of $V$ and elements $x_{i} \in \pi_{n_{i}}(X)$. Define a function $\widetilde{K}: \wedge^{2} V \rightarrow \mathbb{Q}$ as follows: For an element $u \in \wedge^{\geq r} V$, we write

$$
u=\sum_{i_{1} \leq \cdots \leq i_{r}} q_{i_{1} \cdots i_{r}} u_{i_{1}} \cdots u_{i_{r}}+w,
$$

where $w \in \wedge^{\geq r+1} V$ and $q_{i_{1} \cdots i_{r}} \in \mathbb{Q}$. Then $\widetilde{K}$ is defined by

$$
\widetilde{K}(u)=\sum_{i_{1} \leq \cdots \leq i_{r}} q_{i_{1} \cdots i_{r}} K\left(A_{i_{1} \cdots i_{r}}\right),
$$

where $A_{i_{1} \cdots i_{r}}$ is the $r \times r$ matrix whose $(p, q)$-entry is the Sullivan pairing $\left\langle u_{i_{p}}, x_{q}\right\rangle$.

Theorem 6.2. ([1, Theorem 5.4]) Let $X$ be a simply-connected rational space with a minimal model $(\wedge V, d)$. Suppose that the higher order Whitehead product set $\left[x_{1}, \ldots, x_{r}\right] \subset \pi_{N-1}(X)$ is non-empty and that $v \in V$ is an element of degree $N-1$ with $d v \in \wedge^{\geq r} V$. Then, for each $x \in\left[x_{1}, \ldots, x_{r}\right]$,

$$
\langle v, x\rangle=(-1)^{\varepsilon} \tilde{K}(d(v)),
$$

where $\varepsilon=\sum_{i<j} n_{i} n_{j}$.

We are ready to prove the main theorem in this section.

Proof of Theorem 6.1. Let $i:(\wedge V, d) \longmapsto(\wedge V \otimes \wedge Z, \widehat{d})$ be the minimal model for $f$. Since the induced map $f_{*}: \pi_{*}(U) \rightarrow \pi_{*}(X)$ is surjective, it follows that the map $\wedge V \rightarrow H\left(\wedge V \otimes \wedge Z, \widehat{d}_{0}\right)$ induced by $i$ is injective, where $\widehat{d}_{0}$ is the linear part of $\widehat{d}$. This implies that $(\wedge V \otimes \wedge Z, \widehat{d})$ is a minimal model for $U$ ([17, 4.12 Proposition]).

We choose a nonzero element $x \in\left[x_{1}, \ldots, x_{r}\right]$. Since all Whitehead products of order $<r$ vanish in $\pi_{*}(X)$, it follows from [1, Proposition 6.4] that $d\left(x^{*}\right)$ is in $\wedge^{\geq r} V$. With a basis $\left\{u_{i}\right\}$ of $V$, we write $d\left(x^{*}\right)=\sum_{i_{1} \leq \cdots \leq i_{r}} q_{i_{1} \cdots i_{r}} u_{i_{1}} \cdots u_{i_{r}}+w$, where $w \in \wedge^{\geq r+1} V$ and $q_{i_{1} \cdots i_{r}}$ are nonzero in $\mathbb{Q}$. Suppose that there exists an element $x_{i}$ such that $\operatorname{deg} x_{i} \neq \operatorname{deg} u_{j_{l}}$ for any $j_{l}$. Then all the numbers $\left\langle u_{j_{l}}, x_{i}\right\rangle$ in $i$ th column of the matrix $A_{j_{1} \ldots j_{r}}$ are zero for any $j_{1}, \ldots, j_{r}$. From Theorem 6.2 , we have $1=\left\langle x^{*}, x\right\rangle=(-1)^{\varepsilon} \tilde{K}\left(d\left(x^{*}\right)\right)=0$, which is a contradiction. Thus we see that for any $x_{i}$ there exists an element $u_{i_{l}}$ with degree equal to deg $x_{i}$, which occurs in a term of $d\left(x^{*}\right)$ as a factor. Without loss of generality, we can assume that

$$
d\left(x^{*}\right)=\sum_{i_{1} \leq \cdots \leq i_{r}} q_{i_{1} \cdots i_{r}} u_{i_{1}} \cdots u_{i_{r}},
$$

modulo $\wedge^{\geq r+1} V$ with $\operatorname{deg} u_{i_{1}}=\operatorname{deg} x_{i}$ for some $i_{1}$. It follows from [1, Proposition 6.4] that $\widehat{d h} \in \wedge^{\geq}(V \oplus Z)$ for any $h \in \wedge V \otimes \wedge Z=\wedge(V \oplus Z)$ and hence $\left\langle h, \widehat{d}_{*}\left(u_{i_{2}} \cdots u_{i_{r}}\right)_{*}\right\rangle=\left\langle\widehat{d} h,\left(u_{i_{2}} \cdots u_{i_{r}}\right)_{*}\right\rangle=0$. This fact yields that $\widehat{d}_{*}\left(u_{i_{2}} \cdots u_{i_{r}}\right)_{*}=$ 0 . Proposition 4.5 allows us to conclude that the element $x$ is detective with respect to $(U, X ; f)$. By definition, we see that $\operatorname{deg} x_{i} \in I(x)$. This completes the proof.

\section{Proof of Theorem 1.6 And examples}

We begin with the $G$-sequence introduced by Woo and Lee in [41]. Let $X$ be a space with basepoint $x_{0}$ and $U$ a subspace of $X$. Let $i: U \rightarrow X$ denote the 
inclusion map. Then the evaluation map ev $:\left(\mathcal{F}(U, X ; i), \mathcal{F}\left(U, U ; i d_{U}\right)\right) \rightarrow(X, U)$ at $x_{0}$ gives rise to the homomorphism

$$
e v_{\sharp *}: \pi_{*}\left(\mathcal{F}(U, X ; i), \mathcal{F}\left(U, U ; i d_{U}\right)\right) \rightarrow \pi_{*}(X, U) .
$$

The image of $e v_{\sharp n}$ is denoted by $G_{n}^{\operatorname{Rel}}(U, X ; i)$. The $G$-sequence

$$
\cdots \rightarrow G_{n}(U) \stackrel{i_{\sharp}}{\rightarrow} G_{n}(U, X ; i) \stackrel{j_{\sharp}}{\rightarrow} G_{n}^{\mathrm{Rel}}(U, X ; i) \stackrel{\partial}{\rightarrow} G_{n-1}(U) \rightarrow \cdots
$$

is given as a subsequence of the homotopy exact sequence

$$
\cdots \rightarrow \pi_{n}(U) \stackrel{i_{\sharp}}{\rightarrow} \pi_{n}(X) \stackrel{j_{\sharp}}{\rightarrow} \pi_{n}(X, U) \stackrel{\partial}{\rightarrow} \pi_{n-1}(U) \rightarrow \cdots .
$$

We observe that the $G$-sequence is not exact in general; see [23] and [25] for this fact. Note that $G_{*}(U, X ; i)$ and $G_{*}^{\mathrm{Rel}}(U, X ; i)$ are denoted by $G_{*}(X, U)$ and $G_{*}^{\mathrm{Rel}}(X, U)$, respectively in [41]. If $F \stackrel{i}{\rightarrow} X \stackrel{p}{\rightarrow} Y$ is a fibration, then we have a commutative diagram

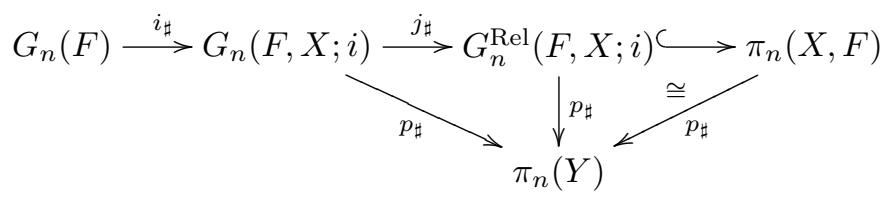

for any $n \geq 2$. The exactness at $G_{n}(F, X ; i)$ of the $G$-sequence is therefore equivalent to that of the sequence

$$
G_{n}(F) \stackrel{i_{\sharp}}{\rightarrow} G_{n}(F, X ; i) \stackrel{p_{\sharp}}{\rightarrow} \pi_{n}(Y) .
$$

After proving Theorem 1.6, we will illustrate non-exactness of the sequence (7.1); see Example 7.5.

In order to prove Theorem 1.6, we need the following result due to Ghorbal [11].

Proposition 7.1. [10, Proposition 3.5] Let $f: X \rightarrow Y$ be a map of rational spaces that admits a minimal model of the form $\gamma:(\wedge(V \oplus W), d) \rightarrow(\wedge W, \bar{d})$ such that $\gamma(V)=0, \gamma(w)=w$ for $w \in W, d(V) \subset \wedge^{2} V \otimes \wedge W$ and $d(W) \subset$ $\wedge W+\wedge \geq 2 V \otimes \wedge W$. Then $f$ is a homotopy monomorphism in the nilpotent category; that is, the induced map of homotopy sets $f_{*}:[A, X] \rightarrow[A, Y]$ is injective whenever $A$ is a nilpotent space.

Proof of Theorem 1.6. By assumption, the projection $p$ admits a Sullivan model $\widetilde{p}:\left(\wedge V_{Y}, d\right) \rightarrow\left(\wedge V_{Y} \otimes \wedge V, \widehat{d}\right)$ such that $\widehat{d}(v) \in \wedge^{\geq 2} V_{Y} \otimes \wedge V+\mathbb{Q} \otimes \wedge^{\geq 2} V$ for any $v \in V$. From $\left[16\right.$, Theorem 20.3], we see that the DGA $(\wedge V, \bar{d})=\mathbb{Q} \otimes\left(\wedge Y_{Y}, d\right)$ $\otimes\left(\wedge V_{Y} \otimes \wedge V, \widehat{d}\right)$ is a minimal model for the fibre $F$. Moreover it follows that the projection $\widetilde{i}: \wedge\left(V_{Y} \oplus V\right) \rightarrow \wedge V$ is a Sullivan representative of $i: F \rightarrow X$. By virtue of Remark 3.4, the 0-simplex $\widetilde{u}_{\tilde{i}} \in \Delta\left(\wedge\left(\left(V_{Y} \oplus V\right) \otimes(\wedge V)_{*}\right)\right)$ gives the Sullivan model $E / M_{u}$ which is a model for $\mathcal{F}(F, X ; i)$, where $u=\Delta(\gamma) \widetilde{u}_{\tilde{i}}$; see Theorem 3.3 and paragraphs before Theorem 2.3 for the notations. Moreover we have a Sullivan model of the form $\left(E^{\prime} / M_{u^{\prime}}^{\prime}, \delta\right)$ for $\mathcal{F}\left(F, F ; i d_{F}\right)$ in which $E^{\prime}=\wedge\left(V \otimes H_{*}(F)\right)$ and $u^{\prime}=\Delta\left(\gamma^{\prime}\right) \widetilde{u}_{i d_{\wedge V}} \in \Delta\left(\wedge\left(V \otimes(\wedge V)_{*}\right)\right)$; see Remark 3.4.

Since the model $\left(\wedge V_{Y} \otimes \wedge V, \widehat{d}\right)$ is minimal, the dual sequence to the homotopy exact sequence of $F \rightarrow X \rightarrow Y$ splits into the short exact sequence $0 \rightarrow V_{Y} \stackrel{Q(\widetilde{p})}{\rightarrow}$ 
$V_{Y} \oplus V \stackrel{Q(\widetilde{i})}{\rightarrow} V \rightarrow 0$. In order to prove Theorem 1.6, it suffices to show that the sequence

is exact.

$$
0 \rightarrow V_{Y} \stackrel{\overline{Q(\widetilde{p})}}{\rightarrow}\left(V_{Y} \oplus V\right) / \operatorname{Ker} Q(\widetilde{e v}) \stackrel{\overline{Q(\widetilde{i})}}{\rightarrow} V / \operatorname{Ker} Q(\widetilde{e v}) \rightarrow 0
$$

Since $F \stackrel{i}{\rightarrow} X \stackrel{p}{\rightarrow} Y$ is a separable fibration, Proposition 7.1 implies that $i$ is a homotopy monomorphism in the category of nilpotent spaces; that is, for any nilpotent space $Z$ and any two maps $u, v: Z \rightarrow F, u \simeq v$ whenever $i u \simeq i v$. The main theorem in [31] asserts that if $i$ is a homotopy monomorphism in based topological spaces, then the $G$-sequence of $(F, X)$ splits into short exact sequences

$$
0 \rightarrow G_{n}(F) \stackrel{i_{\sharp}}{\rightarrow} G_{n}(F, X ; i) \stackrel{j_{\sharp}}{\rightarrow} G_{n}^{\operatorname{Rel}}(F, X ; i) \rightarrow 0 .
$$

The same argument as in the proof of [31, Theorem 2.2] does work well to show that the sequence is exact at $G_{*}(F)$ and $G_{*}(F, X ; i)$. Indeed, in the proof, it is only needed that $i: F \rightarrow X$ is a homotopy monomorphism with respect to $\left(S^{k} \times F\right) /\left(S^{k} \times *\right)$ with $k \geq 2$ in the category $\mathcal{T} O P_{*}$ of based topological spaces; see [31, Lemma 2.1].

Let $[M, N]_{*}$ denote the set of based homotopy classes between based spaces $M$ and $N$. Since $F$ is simply-connected, it follows that $\left(S^{k} \times F\right) /\left(S^{k} \times *\right)$ is simply connected and hence nilpotent. Thanks to the result due to Ghorbal mentioned above, the induced map $i_{*}:\left[\left(S^{k} \times F\right) /\left(S^{k} \times *\right), F\right] \rightarrow\left[\left(S^{k} \times F\right) /\left(S^{k} \times *\right), X\right]$ is injective. We see that the natural map $\left[\left(S^{k} \times F\right) /\left(S^{k} \times *\right), F\right]_{*} \rightarrow\left[\left(S^{k} \times F\right) /\left(S^{k} \times\right.\right.$ $*), F]$ is bijective because $F$ is simply-connected. Therefore the induced map

$$
i_{*}:\left[\left(S^{k} \times F\right) /\left(S^{k} \times *\right), F\right]_{*} \rightarrow\left[\left(S^{k} \times F\right) /\left(S^{k} \times *\right), X\right]_{*}
$$

is injective so that $i: F \rightarrow X$ is a homotopy monomorphism with respect to $\left(S^{k} \times F\right) /\left(S^{k} \times *\right)$ with $k \geq 2$ in $\mathcal{T} O P_{*}$. Thus, we are left to prove that $\overline{Q(\widetilde{p})}$ is a monomorphism.

Recall the model $\left(E / M_{u}, \delta\right)$ for $\mathcal{F}(F, X ; i)$ from Remark 3.4 and DGA maps described in Section 2; see the diagram below.

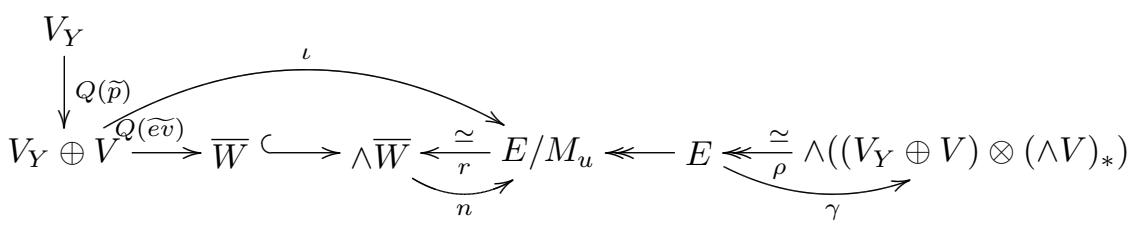

Let $\delta_{0}$ be the linear part of the differential $\delta$. Suppose that $v^{Y}$ is in $\operatorname{Ker} Q(\widetilde{e v})$ for a nonzero element $v^{Y} \in V_{Y} \subset V_{Y} \oplus V$. Choose a basis $\left\{v_{i}\right\}$ of $V_{Y} \oplus V$ so that $v_{Y} \in\left\{v_{i}\right\}$. Lemma 3.5 yields that $\iota\left(v^{Y}\right)=v^{Y} \otimes 1$ is in $\operatorname{Im} \delta_{0}$. Hence the element $v^{Y} \otimes 1$ appears in $\delta_{0}\left(v_{i} \otimes e\right)$ as a summand of a term for some element $v_{i} \otimes e$ in $\left(V_{Y} \oplus V\right) \otimes(\wedge V)_{*}$. Therefore $\widehat{d}\left(v_{i}\right)$ contains $v^{Y}$ as a factor; see Remark 2.6. We write

$$
\widehat{d}\left(v_{i}\right)=\sum_{i_{1}, . ., i_{s}, j_{1}, \ldots, j_{m}} v_{i_{1}}^{Y} \cdots v_{i_{s}}^{Y} \cdot v_{j_{1}} \cdots v_{j_{m}}+\sum_{j_{1}, \ldots, j_{q}} v_{j_{1}}^{\prime} \cdots v_{j_{q}}^{\prime},
$$

where $v_{i_{l}}^{Y} \in V_{Y}, v_{j_{l}}, v_{j_{m}}^{\prime} \in V$. Observe that $s \geq 2$ and $q \geq 2$ because the given fibration $F \rightarrow X \rightarrow Y$ is separable. Without loss of generality, we assume that $v_{i_{1}}^{Y}=v^{Y}$. Then the other element $v_{i_{l}}^{Y}(l \geq 2)$ needs to be an element of $\mathbb{Q}$ after tensoring some element $e^{\prime}$ in $(\wedge V)_{*}$ which appears in $D^{(s+m-1)}(e)$ as a factor; that 
is, $\operatorname{deg} v_{i_{l}}^{Y} \otimes e^{\prime}=0$ and $v_{i_{l}}^{Y} \otimes e^{\prime} \in \mathbb{Q}$ in $E / M_{u}$. However $v_{i_{l}}^{Y} \otimes e^{\prime}=\widetilde{u}_{\tilde{i}}\left(v_{i_{l}}^{Y} \otimes e^{\prime}\right)=0$; see (3.1). In consequence, the element $v^{Y} \otimes 1$ does not appear in $\delta_{0}\left(v_{i} \otimes e\right)$ as a factor, which is a contradiction. This completes the proof.

We here recall from [9, Section 15 (c)] a model for a pullback fibration. Let

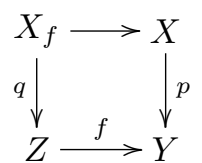

is a pullback diagram in which $Y$ and $Z$ are simply-connected and $p$ is a fibration with fibre $F$. Let $\left(\wedge V_{Y}, d\right) \rightarrow\left(\wedge V_{Z}, d\right)$ be a Sullivan representative for $f$ and

$$
(\wedge W, \bar{d}) \ll \pi
$$

a model for the fibration $p$; that is, there exists a commutative diagram

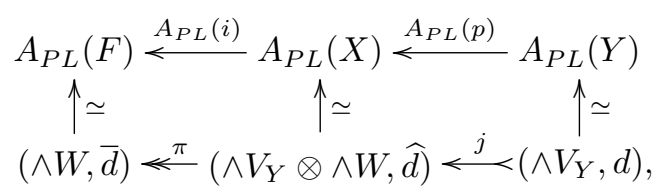

in which vertical arrows are quasi-isomorphisms, $j$ is a Sullivan model for $p$ and $\pi$ is the natural projection.

Proposition 7.2. [9, Proposition 15.8] The pullback fibration $F \rightarrow X_{f} \stackrel{q}{\rightarrow} Z$ has a model of the form $(\wedge W, \bar{d}) \longleftarrow\left(\wedge V_{Z}, d\right) \otimes_{\left(\wedge V_{Y}, d\right)}\left(\wedge V_{Y} \otimes \wedge W, \widehat{d}\right) \longleftrightarrow\left(\wedge V_{Z}, d\right)$.

Example 7.3. Let $F \rightarrow X \stackrel{p}{\rightarrow} Y$ be a fibration over a simply-connected space $Y$ and $f: Z \rightarrow Y$ a map. If the fibration $p: X \rightarrow Y$ is separable, then so is the pullback fibration $q: X_{f} \rightarrow Z$ of $p$ by $f$. This follows from Proposition 7.2.

Examples 7.4 and 7.5 described below show that there is a separable fibration which can be obtained from a non-separable fibration via the pullback construction by an appropriate map.

Example 7.4. Let $G$ be a compact simply connected Lie group with a maximal torus $T$ and $i: T \rightarrow G$ the inclusion. Then the Borel fibration

$$
G / T \stackrel{j}{\rightarrow} E_{T} \times_{T} G / T \stackrel{p}{\rightarrow} B T
$$

is separable. To see this, we consider the fibration $G / T \rightarrow B T \stackrel{B i}{\rightarrow} B G$. Suppose that $H^{*}(B G ; \mathbb{Q}) \cong \mathbb{Q}\left[y_{1}, \ldots, y_{l}\right]$ and $H^{*}(B T ; \mathbb{Q}) \cong \mathbb{Q}\left[t_{1}, \ldots, t_{l}\right]$. Put $(B i)^{*}\left(y_{i}\right)=$ $f_{i}\left(t_{1}, \ldots, t_{l}\right)$. Since $\operatorname{deg} y_{s} \geq 4$ and $\operatorname{deg} t_{s}=2$ for $1 \leq s \leq l$, it follows that $f_{i}\left(t_{1}, \ldots, t_{l}\right)$ is decomposable for any $i$. Moreover we see that the fibration $B i: B T \rightarrow B G$ has a model of the form

$$
\left(\wedge\left(t_{i}\right) \otimes \wedge\left(z_{i}\right), \bar{d}\right) \ll\left(\wedge\left(t_{i}\right) \otimes \wedge\left(z_{i}\right) \otimes \wedge\left(y_{i}\right), \widehat{d}\right) \ll<\left(\wedge\left(y_{i}\right), 0\right),
$$

in which $\widehat{d}\left(z_{i}\right)=-y_{i}+f_{i}\left(t_{1}, \ldots, t_{l}\right)$ for any $i$. The uniqueness of a minimal relative Sullivan algebra ([9, Theorem 14.12]) implies that the fibration $B i: B T \rightarrow B G$ is not separable. 
The fibration $G / T \stackrel{j}{\rightarrow} E_{T} \times_{T} G / T \stackrel{p}{\rightarrow} B T$ fits into the fibre square

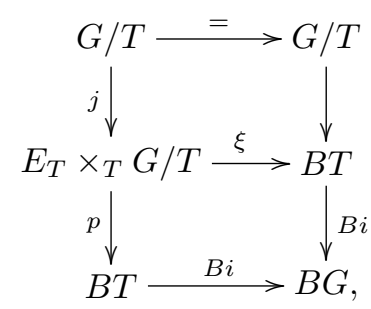

where $\xi: E_{T} \times_{T} G / T \rightarrow B T$ is defined by sending an element $\left[x,[g]_{T}\right]$ to $[x g]_{T}$; see $[20,(2.2)]$. By using Proposition 7.2 , we have a model for the Borel fibration $E_{T} \times_{T} G / T \stackrel{p}{\rightarrow} B T$ of the form

$$
\left(\wedge\left(t_{i}\right) \otimes \wedge\left(z_{i}\right), \bar{d}\right) \ll\left(\wedge\left(t_{i}^{\prime}\right) \otimes \wedge\left(t_{i}\right) \otimes \wedge\left(z_{i}\right), \widehat{d}\right) \longleftarrow\left(\wedge\left(t_{i}^{\prime}\right), 0\right)
$$

where $\widehat{d}\left(z_{i}\right)=-f_{i}\left(t_{1}^{\prime}, \ldots, t_{l}^{\prime}\right)+f_{i}\left(t_{1}, \ldots, t_{l}\right)$ for any $i$. This enables us to deduce that the fibration $E_{T} \times_{T} G / T \stackrel{p}{\rightarrow} B T$ is separable. Moreover, Theorem 1.6 yields that

$$
G_{*}\left(G / T, E_{T} \times_{T} G / T ; j\right) \otimes \mathbb{Q} \cong G_{*}(G / T) \otimes \mathbb{Q} \oplus \pi_{*}(B T) \otimes \mathbb{Q} .
$$

Since $G_{\text {even }}(G / T) \otimes \mathbb{Q}=0$ ([8, Theorem III $\left.]\right)$, it follows that $G_{\text {even }}\left(G / T, E_{T} \times_{T}\right.$ $G / T ; j) \otimes \mathbb{Q} \cong \pi_{\text {even }}(B T) \otimes \mathbb{Q}$ and hence

$$
G_{\text {even }}\left(G / T, E_{T} \times_{T} G / T ; j\right) \otimes \mathbb{Q} \cong \mathbb{Q}\left\{t_{1}^{\prime *}, \ldots, t_{l}^{\prime *}\right\}
$$

where $\left\{t_{1}^{\prime *}, \ldots, t_{l}^{\prime *}\right\}$ is the dual to the basis $\left\{t_{1}^{\prime}, \ldots, t_{l}^{\prime}\right\}$ of the vector space which generates the model for $B T$ mentioned above. Moreover, by virtue of Proposition 2.5 , we have

$$
G_{\text {odd }}\left(G / T, E_{T} \times_{T} G / T ; j\right) \otimes \mathbb{Q} \cong G_{\text {odd }}(G / T) \otimes \mathbb{Q} \cong \pi_{\text {odd }}(G / T) \otimes \mathbb{Q} \cong \mathbb{Q}\left\{z_{i}\right\} .
$$

For a more general result, see [27, Corollary 5.5].

Example 7.5. The fibration $S^{2} \stackrel{i}{\rightarrow} \mathbb{C} P^{3} \stackrel{p}{\rightarrow} S^{4}$ obtained by the Hopf bundle $S^{3} \rightarrow$ $S^{7} \rightarrow S^{4}$ is not separable.

(i) The sequence

$$
G_{*}\left(S^{2}\right) \stackrel{i_{\sharp}}{\rightarrow} G_{*}\left(S^{2}, \mathbb{C} P^{3} ; i\right) \stackrel{p_{\sharp}}{\rightarrow} \pi_{*}\left(S^{4}\right)
$$

is not exact. In fact, we have a model for the fibration $S^{2} \stackrel{i}{\rightarrow} \mathbb{C} P^{3} \stackrel{p}{\rightarrow} S^{4}$ of the form

$$
\left(\wedge\left(x_{2}, x_{3}\right), d x_{3}=x_{2}^{2}\right) \stackrel{\pi}{\longleftarrow}\left(\wedge\left(x_{2}, x_{3}, y_{4}, y_{7}\right), \widehat{d}\right) \longleftrightarrow\left(\wedge\left(y_{4}, y_{7}\right), d y_{7}=y_{4}^{2}\right),
$$

where the differential $\widehat{d}$ is defined by $\widehat{d} y_{7}=y_{4}^{2}$ and $\widehat{d} x_{3}=x_{2}^{2}+y_{4}$. Since $S^{2}$ is a finite complex, the sequence

$$
G_{*}\left(S_{\mathbb{Q}}^{2}\right) \stackrel{i_{\sharp}}{\rightarrow} G_{*}\left(S_{\mathbb{Q}}^{2}, \mathbb{C} P_{\mathbb{Q}}^{3} ; i\right) \stackrel{p_{\sharp}}{\rightarrow} \pi_{*}\left(S_{\mathbb{Q}}^{4}\right)
$$

is regarded as that obtained tensoring $\mathbb{Q}$ to $(7.2)$; see [35, Theorem 2.3]. Thus in order to prove non-exactness of the sequence (7.2), it suffices to show that the dual sequence to $(7.3)$

$$
V \rightarrow H\left(V \oplus W, \widehat{d}_{0}\right) / \operatorname{Ker} Q(\widetilde{e v}) \stackrel{\overline{Q(\widetilde{i})}}{\rightarrow} W / \operatorname{Ker} Q(\widetilde{e v})
$$


is not exact, where $V=\mathbb{Q}\left\{y_{4}, y_{7}\right\}, W=\mathbb{Q}\left\{x_{2}, x_{3}\right\}$ and $\widehat{d}_{0}\left(x_{3}\right)=y_{4}$. Observe that $H\left(V \oplus W, \widehat{d}_{0}\right) \cong \mathbb{Q}\left\{x_{2}, y_{7}\right\}$. We have a quasi-isomorphism

$$
m:\left(\wedge\left(x_{2}, y_{7}\right), d y_{7}=x_{2}^{4}\right) \stackrel{\simeq}{\longrightarrow}\left(\wedge\left(x_{2}, x_{3}, y_{4}, y_{7}\right), \widehat{d}\right)
$$

which sends $x_{2}$ and $y_{7}$ to $x_{2}$ and $y_{7}-y_{4} x_{3}+x_{2}^{2} x_{3}$, respectively. Therefore the map $\pi \circ m$ is a Sullivan representative for the inclusion $i: S^{2} \rightarrow \mathbb{C} P^{3}$. The element $x_{2} \in H\left(V \oplus W, \widehat{d}_{0}\right) / \operatorname{Ker} Q(\widetilde{e v})$ maps to zero by $\overline{Q(i)}$ because $\widetilde{e v}\left(x_{2}\right)=x_{2} \otimes 1$ coincides with the image $\delta_{0}\left(\frac{1}{2}\left(x_{3} \otimes x_{2 *}\right)\right)$ in the model for $\mathcal{F}\left(S_{\mathbb{Q}}^{2}, S_{\mathbb{Q}}^{2} ; i d\right)$. Suppose that (7.3) is exact. Since there is no element with degree 2 in $V$, it follows that $Q(\widetilde{e v})\left(x_{2}\right)=0$ for the linear part $Q(\widetilde{e v})$ of the model $\widetilde{e v}$ for the evaluation map $e v: \mathcal{F}\left(S_{\mathbb{Q}}^{2}, \mathbb{C} P_{\mathbb{Q}}^{3} ; i\right) \rightarrow \mathbb{C} P_{\mathbb{Q}}^{3}$. By virtue of Lemma 3.5 , there is an element $w \in E / M_{u}$ such that $\delta_{0}(w)=x_{2} \otimes 1=\widetilde{e v}\left(x_{2}\right)$, where $E \cong \wedge\left(\mathbb{Q}\left\{x_{2}, y_{7}\right\} \otimes H_{*}\left(S^{2}\right)\right)$ and $u=$ $\Delta(\gamma) \widetilde{u}_{\pi \circ m}$. For dimensional reasons, there is no element with degree 1 in $E / M_{u}$, which is a contradiction.

(ii) Let $q: S^{2} \times S^{2} \rightarrow S^{2} \times S^{2} / S^{2} \vee S^{2} \cong S^{4}$ be the projection and $S^{2} \stackrel{i}{\rightarrow} X \stackrel{\pi}{\rightarrow} S^{2} \times S^{2}$ the pullback by $q$ of the fibration $S^{2} \stackrel{i}{\rightarrow} \mathbb{C} P^{3} \stackrel{p}{\rightarrow} S^{4}$. By applying Proposition 7.2 to the model for the fibration $S^{2} \stackrel{i}{\rightarrow} \mathbb{C} P^{3} \stackrel{p}{\rightarrow} S^{4}$ mentioned in (i), we see that $\pi$ is separable. It follows from Theorem 1.6 that

$$
\begin{aligned}
G_{n}\left(S_{\mathbb{Q}}^{2}, X_{\mathbb{Q}} ; i_{\mathbb{Q}}\right) & \cong G_{n}\left(S_{\mathbb{Q}}^{2}\right) \oplus \pi_{n}\left(S_{\mathbb{Q}}^{2} \times S_{\mathbb{Q}}^{2}\right) \\
& \cong \begin{cases}\mathbb{Q} \oplus \mathbb{Q} & \text { if } n=2 \\
\mathbb{Q} \oplus \mathbb{Q} \oplus \mathbb{Q} & \text { if } n=3 \\
0 & \text { otherwise }\end{cases}
\end{aligned}
$$

We conclude this section with a result concerning the group $G_{*}^{\mathrm{Rel}}(F, X ; i)$, which is deduced by combining Theorem 1.6 with [31, Theorem 2.2].

Corollary 7.6. Under the same assumptions as in Theorem 1.6, the induced map

$$
p_{*}: G_{n}^{\mathrm{Rel}}(F, X ; i) \rightarrow \pi_{n}(Y)
$$

is an isomorphism for $n>1$.

\section{The evaluation subgroup of the Fundamental group}

In order to prove Theorem 1.7, we first recall a filtration of a Sullivan algebra. Let $A=(\wedge W, d)$ be a Sullivan algebra and put $A(-1)=A^{0}=\mathbb{Q}$. Let $A(n)$ denote the sub DGA generated by $A^{i}$ for $0 \leq i \leq n$ and $d A^{n}$. Define $A(n, q) \subset A(n)$ to be the sub DGA generated by $A(n, q-1)$ and the set $\left\{a \in A^{n} \mid d a \in A(n, q-1)\right\}$, where $A(n, 0)=A(n-1)$. Observe that $A(1,0)=\mathbb{Q}$ and that $d y=0$ for any $y \in A(1,1)$. As usual, for an augmentation algebra $C$, let $Q(C)$ denote the vector space of indecomposable elements, namely, $Q(C)=\bar{C} / \bar{C} \cdot \bar{C}$, where $\bar{C}$ denotes the augmentation ideal. The result $[2,12.7$ Theorem] asserts that for any connected nilpotent space $X$ with a Sullivan model $\wedge W$ of finite type, there exists a natural isomorphism

$$
\left(\Gamma^{q+1} / \Gamma^{q}\right) \pi^{1}(\wedge W) \cong \operatorname{Hom}_{\mathbb{Z}}\left(\left(\Gamma_{q} / \Gamma_{q+1}\right) \pi_{1}(X), \mathbb{Q}\right),
$$

where $\Gamma^{i} \pi^{1}(\wedge W)$ is the image of the induced map $\pi^{1}(A(1, i-1)) \rightarrow \pi^{1}(A)$ by the natural inclusion and $\left(\Gamma^{q+1} / \Gamma^{q}\right) \pi^{1}(\wedge W)$ denotes the subquotient $\Gamma^{q+1} \pi^{1}(\wedge W) / \Gamma^{q} \pi^{1}(\wedge W)$. By using this result, we prove Theorem 1.7. 
Proof of Theorem 1.7. We first observe that the diagram (1.1) in the Introduction gives rise to the diagram

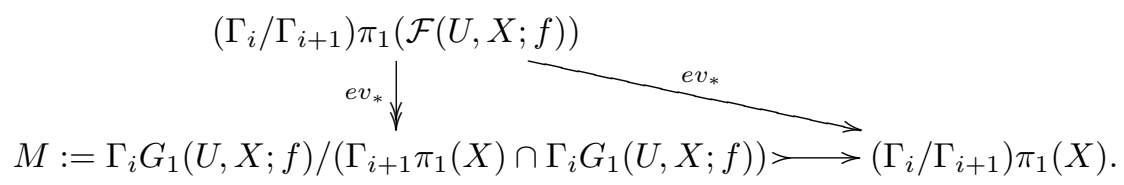

Let $(\wedge W, d)$ be a minimal model for $X$. When $U$ is a finite CW complex, it follows from [19, Theorem 3.11] that $e_{X *}: \mathcal{F}(U, X ; f) \rightarrow \mathcal{F}\left(U, X_{\mathbb{Q}} ; e_{X} \circ f\right)$ is a localization. Therefore with the hypothesis (ii), we see that $\left(E / M_{u}, \delta\right)$ described in Section 3 is a Sullivan model for $\mathcal{F}(U, X ; f)$. By dualizing the diagram above, we have a commutative diagram $(* *)_{i}$ :

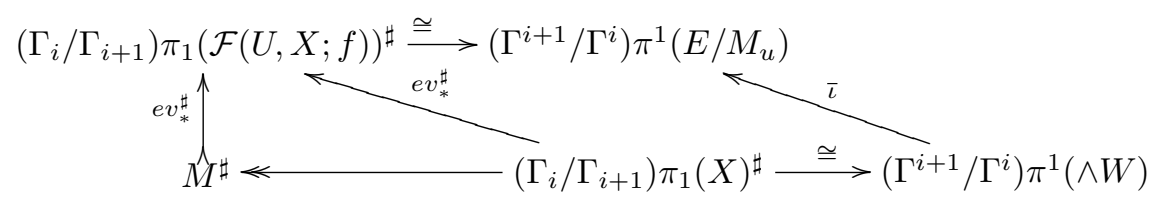

in which $\bar{\iota}$ is the linear map naturally induced by the model $\iota$ for the evaluation map $e v$ in Theorem 3.3. Since $(\wedge W, d)$ is minimal, it follows that

$$
\Gamma^{i+1} \pi^{1}(\wedge W)=\operatorname{Im}\{Q(\wedge W(1, i)) \rightarrow W\}=Q(\wedge W(1, i)) .
$$

(1) We observe that the differential on elements of degree 1 is strictly quadratic for degree reasons, since $A^{0}=\mathbb{Q}$.

Since $\left(\Gamma_{k} / \Gamma_{k+1}\right) \pi_{1}(X)^{\sharp} \neq 0$, it follows that $\left(\Gamma^{i+1} / \Gamma^{i}\right) \pi^{1}(\wedge W) \neq 0$ for any $i \leq k$. For any $i<k$, let $\left\{y_{j}\right\}_{j \geq 1}$ be the basis of $Q(\wedge W(1, i))$ for which $y_{1}, \ldots, y_{m}$ are linearly independent in the vector space $Q(\wedge W(1, i)) / Q(\wedge W(1, i-1))$. Then there exists an element $y^{(i+1)} \in\left(\Gamma^{i+2} / \Gamma^{i+1}\right) \pi^{1}(\wedge W)$ such that

$$
\begin{aligned}
d y^{(i+1)}= & c_{1} y_{i_{1}} y_{\alpha_{1}}+c_{2} y_{i_{1}} y_{\alpha_{2}}+\cdots+c_{k} y_{i_{1}} y_{\alpha_{k}} \\
& +c_{k+1} y_{i_{k+1}} y_{\alpha_{k+1}}+\cdots+c_{m} y_{i_{m}} y_{\alpha_{m}}+\cdots+c_{s} y_{i_{s}} y_{\alpha_{s}}+w,
\end{aligned}
$$

where $c_{i} \neq 0,1 \leq i_{1} \leq m, \alpha_{n} \neq i_{1}$ for $1 \leq n \leq k, \alpha_{i} \neq \alpha_{j}$ for $i, j \leq k$ with $i \neq j, i_{1} \neq i_{q}$ for $\bar{k}+1 \leq q \leq s, y_{i_{q}} y_{\alpha_{q}} \neq y_{i_{r}} y_{\alpha_{r}}$ if $q \neq r$ and $w \in Q(\wedge W(1, i-1))$. $Q(\wedge W(1, i-1))$. Using the element $y^{(i+1)}$, we can show, as in the proof of Theorem 4.2 , that $\iota$ is not a monomorphism. In fact, let $(\wedge W, d) \longmapsto(\wedge W \otimes \wedge Z, \widehat{d})=: B$ be the Sullivan model for the given map $f$ with which we construct the model $\left(E / M_{u}, \delta\right)$ for $\mathcal{F}(U, X ; f)$. It follows from the hypothesis (i) and the minimality of the Sullivan model for $f$ with $(4.1)$ that $\widehat{d}_{*}\left(\left(y_{j}\right)_{*}\right)=0$ for any $j$ in the differential coalgebra $\left((\wedge W \otimes \wedge Z)_{*}, \widehat{d}_{*}\right)$. Proposition 4.5 enables us to conclude that $y^{(i+1)}$ is a detective element with respect to $(U, X ; f)$. Therefore, we see that in $\wedge\left(W \otimes B_{*}\right) / M_{\widetilde{u}}$

$\delta\left(y^{(i+1)} \otimes\left(y_{\alpha_{1}}\right)_{*}\right)=-c_{1} y_{i_{1}} \otimes 1-\sum_{j \geq k+1, y_{\alpha_{j}}=y_{\alpha_{1}}} c_{j} y_{i_{j}} \otimes 1+\sum_{j \geq k+1, y_{i_{j}}=y_{\alpha_{1}}} c_{j} y_{\alpha_{j}} \otimes 1+v \otimes 1$

for some element $v \in Q(\wedge W(1, i-1))$ in $\wedge\left(W \otimes B_{*}\right) / M_{\widetilde{u}}$; see Lemma 4.3. Put

$$
w=-c_{1} y_{i_{1}}-\sum_{j \geq k+1, y_{\alpha_{j}}=y_{\alpha_{1}}} c_{j} y_{i_{j}}+\sum_{j \geq k+1, y_{i_{j}}=y_{\alpha_{1}}} c_{j} y_{\alpha_{j}}+v .
$$

Then the element $w \otimes 1$ is zero in $\Gamma^{i+1} \pi^{1}\left(E / M_{u}\right)$. As in the proof of Theorem 4.2, we see that $\bar{\iota}(w)=0$. This yields that $\operatorname{dim} M^{\sharp} \leq \operatorname{dim}\left(\Gamma_{i} / \Gamma_{i+1}\right) \pi_{*}(X)^{\sharp}-1$. We 
have the result.

(2) Let $\left\{y_{q}\right\}$ be a basis of $\wedge W(1,1)=\Gamma^{2}(\wedge W)$. Since $\left(\Gamma_{2} / \Gamma_{3}\right) \pi_{1}(X)^{\sharp} \neq 0$, there exists an element $y \in\left(\Gamma^{3} / \Gamma^{2}\right) \pi^{1}(\wedge W)$ such that

$$
\begin{aligned}
d y= & c_{1} y_{i_{1}} y_{\alpha_{1}}+c_{2} y_{i_{1}} y_{\alpha_{2}}+\cdots+c_{k} y_{i_{1}} y_{\alpha_{k}} \\
& +c_{k+1} y_{i_{k+1}} y_{\alpha_{k+1}}+\cdots+c_{m} y_{i_{m}} y_{\alpha_{m}}+\cdots+c_{s} y_{i_{s}} y_{\alpha_{s}},
\end{aligned}
$$

where $c_{i} \neq 0, \alpha_{i} \neq \alpha_{j}$ if $i \neq j$ for $i, j \leq k, \alpha_{l} \neq i_{1}$ for $1 \leq l \leq k, y_{i_{q}} y_{\alpha_{q}} \neq y_{i_{r}} y_{\alpha_{r}}$ if $q \neq r$, and $i_{1} \neq i_{m}$ for $m \geq k+1$. The same argument as in the proof of (1) yields that $y$ is a detective element. Thus in $\left(\wedge\left(W \otimes B_{*}\right) / M_{\widetilde{u}}, \delta\right)$, we see that $\delta\left(y \otimes\left(y_{i_{1}}\right)_{*}\right)=c_{1} y_{\alpha_{1}} \otimes 1+\cdots+c_{k} y_{\alpha_{k}} \otimes 1$ and

$$
\delta\left(y \otimes\left(y_{\alpha_{1}}\right)_{*}\right)=-c_{1} y_{i_{1}} \otimes 1-\sum_{m \geq k+1, y_{\alpha_{m}}=y_{\alpha_{1}}} c_{m} y_{i_{m}} \otimes 1+\sum_{m \geq k+1, y_{i_{m}}=y_{\alpha_{1}}} c_{m} y_{\alpha_{m}} \otimes 1 .
$$

It is immediate that the elements $w_{1}=c_{1} y_{\alpha_{1}}+\cdots+c_{k} y_{\alpha_{k}}$ and

$$
w_{2}=-c_{1} y_{i_{1}}-\sum_{m \geq k+1, y_{\alpha_{m}}=y_{\alpha_{1}}} c_{m} y_{i_{m}}+\sum_{m \geq k+1, y_{i_{m}}=y_{\alpha_{1}}} c_{m} y_{\alpha_{m}}
$$

are linearly independent in $\Gamma^{2}(\wedge W)$. Moreover it follows that $\bar{\iota}\left(w_{1}\right)=0=\bar{\iota}\left(w_{2}\right)$ in $\left(\Gamma^{2} / \Gamma^{1}\right) \pi^{1}\left(E / M_{u}\right)$. This completes the proof.

Proof of Theorem 1.9. Let $S^{1} \rightarrow X_{f} \rightarrow T^{n}$ be the $S^{1}$-bundle with the the classifying map $f$ represented by $\rho_{f}=\sum_{i<j} c_{i j} t_{i} t_{j}$ in $H^{2}\left(T^{n} ; \mathbb{Z}\right) \cong\left[T^{n}, K(\mathbb{Z}, 2)\right]$. It follows from the proof of $\left[37\right.$, Proposition (1)] that $X_{f}$ has the homotopy type of a finite CW complex.

The short exact sequence $0 \rightarrow \mathbb{Z} \rightarrow \pi_{1}\left(X_{f}\right) \rightarrow \mathbb{Z}^{\oplus n} \rightarrow 0$ derived from the fibration $S^{1} \rightarrow X_{f} \rightarrow T^{n}$ enables us to conclude that $\pi_{1}\left(X_{f}\right)$ is finitely generated and has no elements of finite order. Thus, $G_{1}\left(X_{f}\right)$ is a finitely generated free abelian group. In order to prove Theorem 1.9 , it suffices to show that $\operatorname{dim} G_{1}\left(X_{f}\right) \otimes \mathbb{Q}=$ $1+n-\operatorname{rank} A_{f}$. To this end, we begin by constructing a minimal model for $X_{f}$.

There exists a fibration of the form $X_{f} \rightarrow T^{n} \stackrel{f}{\rightarrow} K(\mathbb{Z}, 2)$. Therefore by virtue of [19, II Theorem 2.9], we see that $X_{f}$ is nilpotent. Moreover we have a commutative diagram

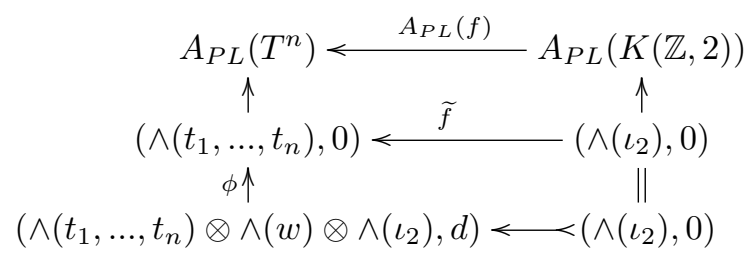

in which vertical arrows in the top square are quasi-isomorphisms, $\tilde{f}$ is a DGA map defined by $\widetilde{f}\left(\iota_{2}\right)=\rho_{f}, d(w)=\rho_{f}-\iota_{2}$ and $\phi(w)=0$. Observe that $\phi$ is also a quasiisomorphism. Thanks to $[9$, Theorem 15.3$]$, the DGA $(\wedge W, \bar{d})=\left(\wedge\left(t_{1}, \ldots, t_{n}\right) \otimes\right.$ $\wedge(w), \bar{d})$ with $\bar{d}(w)=\rho_{f}$ is a minimal model for $X_{f}$.

To simplify, we write $\Gamma^{q+1} / \Gamma^{q}$ and $\Gamma_{q} / \Gamma_{q+1}$ for the subquotients $\left(\Gamma^{q+1} / \Gamma^{q}\right) \pi^{1}(\wedge W)$ and $\left(\Gamma_{q} / \Gamma_{q+1}\right) \pi_{1}\left(X_{f}\right)$, respectively. It follows that $H^{1}\left(X_{f} ; \mathbb{Q}\right)$ is the $n$-dimensional vector space with basis $\left\{t_{1}, t_{2} \ldots, t_{n}\right\}$. Furthermore we have $\Gamma^{2} \pi^{1}(\wedge W)=\mathbb{Q}\left\{t_{1}, \ldots, t_{n}\right\}$ and $\Gamma^{3} \pi^{1}(\wedge W)=\mathbb{Q}\left\{t_{1}, \ldots, t_{n}, w\right\}$.

Recall the DGA $\left(E / M_{u}, \delta\right)$ described in Section 3, where $u \in \Delta(E)$ is the 0 simplex which is induced by the 0 -simplex $\widetilde{u}$ of $\Delta\left(\wedge\left(W \otimes B_{*}\right)\right)$ defined by $\widetilde{u}\left(a \otimes b_{*}\right)=$ 
$(-1)^{\alpha(|a|)} b_{*}(a)$; see Theorem 3.3. Since $X_{f}$ is a finite CW complex, the DGA $\left(E / M_{u}, \delta\right)$ is a Sullivan model for $\mathcal{F}\left(X_{f}, X_{f} ; i d\right)$. Thus we also have the same diagram $(* *)_{1}$ as in the proof of Theorem 1.7.

We compute the image of the element $w \otimes\left(t_{k}\right)_{*}$ by the differential $\delta$ in $E / M_{u}$. From the definition of $\delta$, we see that

$$
\begin{aligned}
\delta\left(w \otimes\left(t_{k}\right)_{*}\right) & =\sum_{i<j} c_{i j} t_{i} t_{j} \otimes\left(t_{k}\right)_{*} \\
& =\left(\sum_{k<j} c_{k j} t_{k} t_{j}+\sum_{i<k} c_{i k} t_{i} t_{k}+\sum_{i<j ; i, j \neq k} c_{i j} t_{i} t_{j}\right)\left(t_{k *} \otimes 1+1 \otimes t_{k *}\right) \\
& =\sum_{k<j} c_{k j} t_{j} \otimes 1-\sum_{i<k} c_{i k} t_{i} \otimes 1 \\
& =\sum_{1 \leq i \leq n ; i \neq k} c_{k i}^{\prime} t_{i} \otimes 1 .
\end{aligned}
$$

We write $u_{k}=\sum_{1 \leq i \leq n ; i \neq k} c_{k i}^{\prime} t_{i}$. Suppose that $\operatorname{rank} A_{f}=m$. Then there exist elements $u_{k_{1}}, \ldots, u_{k_{m}}$ which are linearly independent in $\Gamma^{2}(\wedge W)=\mathbb{Q}\left\{t_{1}, \ldots, t_{n}\right\}$.

Claim 8.1. $\pi_{1}\left(X_{f}\right)$ is not abelian and $\operatorname{dim}\left(\left[\pi_{1}\left(X_{f}\right), \pi_{1}\left(X_{f}\right)\right] \cap G_{1}\left(X_{f}\right)\right) \otimes \mathbb{Q}=1$.

Claim 8.2. Ker $\bar{\iota}=\mathbb{Q}\left\{u_{k_{1}}, \ldots, u_{k_{m}}\right\}$.

Thus we have $\operatorname{dim} M^{\sharp}=n-\operatorname{dim} \operatorname{Ker} \bar{\imath}=n-\operatorname{rank} A_{f}$. It follows from Claim 8.1 that $\operatorname{dim} G_{1}\left(X_{f}\right) \otimes \mathbb{Q}=\operatorname{dim}\left(\left[\pi_{1}\left(X_{f}\right), \pi_{1}\left(X_{f}\right)\right] \cap G_{1}\left(X_{f}\right)\right) \otimes \mathbb{Q}+n-\operatorname{rank} A_{f}=$ $1+n-\operatorname{rank} A_{f}$. We have the result.

Proof of Claim 8.1. We first show that $\pi_{1}\left(X_{f}\right)$ is not abelian. Recall from [19, Proposition 1.10] that the localization functor is exact. Therefore the lower central series of $\pi_{1}\left(X_{f}\right)$ gives rise to a sequence of inclusions

$$
\pi_{1}\left(X_{f}\right)_{\mathbb{Q}}=\Gamma_{1 \mathbb{Q}} \supset \Gamma_{2 \mathbb{Q}} \supset \cdots \supset \Gamma_{j \mathbb{Q}} \supset \cdots .
$$

Moreover we see that $\left(\Gamma_{i} / \Gamma_{i+1}\right) \otimes \mathbb{Q} \cong \Gamma_{i \mathbb{Q}} / \Gamma_{i+1 \mathbb{Q}}$. Thus it follows that for $i \geq 3$

$$
\operatorname{Hom}_{\mathbb{Q}}\left(\Gamma_{i} / \Gamma_{i+1} \otimes \mathbb{Q}, \mathbb{Q}\right) \cong \operatorname{Hom}_{\mathbb{Z}}\left(\Gamma_{i} / \Gamma_{i+1}, \mathbb{Q}\right) \cong \Gamma^{i+1} / \Gamma^{i}=0
$$

and hence $\Gamma_{i \mathbb{Q}}=\Gamma_{i+1 \mathbb{Q}}=\{1\}$ for $i \geq 3$. The existence of an isomorphism between $\Gamma_{2 \mathbb{Q}}$ and $\left(\Gamma_{2} / \Gamma_{3}\right) \otimes \mathbb{Q}$ yields that $\left(\Gamma_{2 \mathbb{Q}}\right)^{\sharp} \cong \operatorname{Hom}_{\mathbb{Z}}\left(\left(\Gamma_{2} / \Gamma_{3}\right) \otimes \mathbb{Q}, \mathbb{Q}\right) \cong$ $\operatorname{Hom}_{\mathbb{Z}}\left(\Gamma_{2} / \Gamma_{3}, \mathbb{Q}\right) \cong \Gamma^{3} / \Gamma^{2} \cong \mathbb{Q}\{w\}$. Thus we see that $\pi_{1}(X)$ is not abelian.

The localized monomorphism

$$
\left(\left[\pi_{1}\left(X_{f}\right), \pi_{1}\left(X_{f}\right)\right] \cap G_{1}\left(X_{f}\right)\right) \otimes \mathbb{Q} \rightarrow\left[\pi_{1}\left(X_{f}\right), \pi_{1}\left(X_{f}\right)\right]_{\mathbb{Q}}=\Gamma_{2 \mathbb{Q}}
$$

induces the epimorphism $\left(\Gamma_{2 \mathbb{Q}}\right)^{\sharp} \rightarrow\left(\left[\pi_{1}\left(X_{f}\right), \pi_{1}\left(X_{f}\right)\right] \cap G_{1}\left(X_{f}\right)\right) \otimes \mathbb{Q}^{\sharp}$. It is readily seen that the dimension of the vector space $\left[\pi_{1}\left(X_{f}\right), \pi_{1}\left(X_{f}\right)\right] \cap G_{1}\left(X_{f}\right) \otimes \mathbb{Q}$ is less than or equal to 1 .

Consider the lower central series of $\pi_{1}\left(X_{f}\right)$ again:

$$
\pi_{1}\left(X_{f}\right)=\Gamma_{1} \supset \Gamma_{2} \supset \cdots \supset \Gamma_{s} \supset \Gamma_{s+1}=\{1\},
$$

where $s=\operatorname{nil}\left(\pi_{1}\left(X_{f}\right)\right)$. Then $\Gamma_{s}$ is a nontrivial abelian subgroup contained in $\mathcal{Z} \pi_{1}\left(X_{f}\right)$, the center of $\pi_{1}\left(X_{f}\right)$. The result [12, Corollary I.13] implies that $\mathcal{Z} \pi_{1}\left(X_{f}\right)=$ $G_{1}\left(X_{f}\right)$ since $X_{f}$ is aspherical, namely, $\pi_{i}\left(X_{f}\right)=0$ for $i>1$. Thus we have $\left[\pi_{1}\left(X_{f}\right), \pi_{1}\left(X_{f}\right)\right] \cap G_{1}\left(X_{f}\right) \supset \Gamma_{s} \neq\{1\}$. As mentioned above, $\pi_{1}\left(X_{f}\right)$ is finitely generated and has no elements of finite order. In particular, $\Gamma_{s}$ is a finitely generated 
free abelian group. This fact yields that $\operatorname{dim}\left(\left[\pi_{1}\left(X_{f}\right), \pi_{1}\left(X_{f}\right)\right] \cap G_{1}\left(X_{f}\right)\right) \otimes \mathbb{Q} \geq 1$. We have the result.

Proof of Claim 8.2. We first observe that the natural projection $E \rightarrow E / M_{u}$ induces an epimorphism $Q(E)^{1}=\mathbb{Q}\left\{t_{i} \otimes 1\right\}_{1 \leq i \leq n} \oplus \mathbb{Q}\{w \otimes 1\} \rightarrow Q\left(E / M_{u}\right)^{1}$. The computation of $\delta\left(w \otimes\left(t_{k}\right)_{*}\right)$ in the proof of Theorem 1.9 allows us to conclude that

$$
Q\left(E / M_{u}\right)^{1}=\left(\mathbb{Q}\left\{t_{i} \otimes 1\right\}_{1 \leq i \leq n} / \mathbb{Q}\left\{u_{k_{j}} \otimes 1\right\}_{1 \leq j \leq m}\right) \oplus \mathbb{Q}\{w \otimes 1\} .
$$

Since $\delta(w \otimes 1)$ is decomposable, it follows that $\pi^{1}\left(E / M_{u}\right)=Q\left(E / M_{u}\right)^{1}$. Moreover we see that

$\pi^{1}\left(\left(E / M_{u}\right)(1,1)\right)=H^{1}\left(Q\left(\left(E / M_{u}\right)(1,1)\right), \delta_{0}\right)=\mathbb{Q}\left\{t_{i} \otimes 1\right\}_{1 \leq i \leq n} / \mathbb{Q}\left\{u_{k_{j}} \otimes 1\right\}_{1 \leq j \leq m}$.

By definition, there exists an epimorphism $\left.\pi^{1}\left(\left(E / M_{u}\right)(1,1)\right)\right) \rightarrow \Gamma^{2} \pi^{1}\left(E / M_{u}\right)$, which is induced by the inclusion $\left(E / M_{u}\right)(1,1) \rightarrow E / M_{u}$. Therefore, we have $\Gamma^{2} \pi^{1}\left(E / M_{u}\right) \cong \mathbb{Q}\left\{t_{i} \otimes 1\right\}_{1 \leq i \leq n} / \mathbb{Q}\left\{u_{k_{j}} \otimes 1\right\}_{1 \leq j \leq m}$. It turns out that

$$
\operatorname{Ker}\left\{\bar{\iota}: \Gamma^{2} \pi^{1}(\wedge W) \rightarrow \Gamma^{2} \pi^{1}\left(E / M_{u}\right)\right\}=\mathbb{Q}\left\{u_{k_{j}}\right\}_{1 \leq j \leq m} .
$$

We proceed to consideration of the rational Gottlieb group in a non-aspherical case. Let $Y$ be the space which admits the Postnikov system of the form

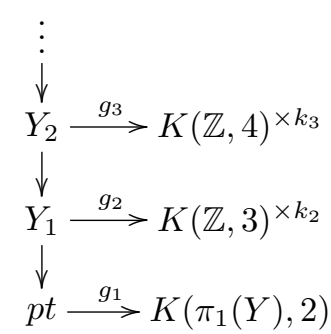

in which $Y_{1}$ is the aspherical space defined to be the pullback of the path-loop fibration $K(\mathbb{Z}, 1)^{\times k} \rightarrow P K(\mathbb{Z}, 2)^{\times k} \rightarrow K(\mathbb{Z}, 2)^{\times k}$ by a map $g: K(\mathbb{Z}, 1)^{\times n} \rightarrow K(\mathbb{Z}, 2)^{\times k}$. We write $g=f_{1} \times \cdots \times f_{k}$ with maps $f_{i}: K(\mathbb{Z}, 1)^{\times n} \rightarrow K(\mathbb{Z}, 2)$ and define the $(n \times n)$-matrix $A_{f_{i}}$ for $1 \leq i \leq k$ as in the paragraph before Theorem 1.9. Let $A_{g}$ be the $(n k \times n)$-matrix consisting of matrices $A_{f_{i}}$. Then the same argument as in the proof of Theorem 1.9 allows us to establish the following theorem.

Theorem 8.3. $\operatorname{dim} G_{1}\left(Y_{\mathbb{Q}}\right) \leq k+n-\operatorname{rank} A_{g}$.

Corollary 8.4. $k \leq \operatorname{rank} G_{1}\left(Y_{1}\right) \leq k+n-\operatorname{rank} A_{g}$.

Proof. We have a $T^{k}$-bundle $T^{k} \stackrel{i}{\rightarrow} Y_{1} \rightarrow T^{n}$. Therefore the induced map $i_{*}$ : $\pi_{1}\left(T^{k}\right) \rightarrow \pi_{1}\left(Y_{1}\right)$, which is a monomorphism, goes through the Gottlieb group $G_{1}\left(Y_{1}\right) \subset \pi_{1}\left(Y_{1}\right)$. The same argument as in the proof of Theorem 1.9 yields that $G_{1}\left(Y_{1}\right)$ is a finitely generated free abelian group. Thus we see that $k \leq \operatorname{rank} G_{1}\left(Y_{1}\right)$. By combining the fact with Theorem 8.3, we have the result since $Y_{1}$ has the homotopy type of a finite CW complex.

Example 8.5. With the same notations as in the proof of Theorem 1.9, let $f$ : $T^{2} \rightarrow K(\mathbb{Z}, 2)$ be the map which represents the element $t_{1} t_{2} \in H^{2}\left(T^{2}, \mathbb{Z}\right)$. Let $K(\mathbb{Z}, 3) \rightarrow Y \rightarrow X_{f} \times S^{3}$ be the pullback fibration of the path-loop fibration 
$K(\mathbb{Z}, 3) \rightarrow P \rightarrow K(\mathbb{Z}, 4)$ by the map $g: X_{f} \times S^{3} \rightarrow K(\mathbb{Z}, 4)$ which represents the element $t_{1} u$, where $u$ is the generator of $H^{3}\left(S^{3} ; \mathbb{Z}\right)$. We now compute the rational Gottlieb group $G_{1}\left(Y_{\mathbb{Q}}\right)$.

We observe that $Y$ is nilpotent space and has a minimal model of the form $A=\left(\wedge\left(t_{1}, t_{2}, w, u, v\right), d\right)$ in which $d(w)=t_{1} t_{2}$ and $d(v)=t_{1} u$. Moreover it follows that $\Gamma^{2} A=\mathbb{Q}\left\{t_{1}, t_{2}\right\}$ and $\Gamma^{3} A=\mathbb{Q}\left\{t_{1}, t_{2}, w\right\}$. The same argument as the proof of Claim 8.1 allows us to deduce that $\operatorname{dim}\left[\pi_{1}\left(Y_{\mathbb{Q}}\right), \pi_{1}\left(Y_{\mathbb{Q}}\right)\right] \cap G_{1}\left(Y_{\mathbb{Q}}\right) \otimes \mathbb{Q} \leq 1$. Since $Y$ is not aspherical, we cannot deduce the same equality as in Claim 8.1 applying [12, Corollary I.13]. However, by virtue of Corollary 1.8, we have

$$
\operatorname{dim} G_{1}\left(Y_{\mathbb{Q}}\right)=\operatorname{dim} G_{1}\left(Y_{\mathbb{Q}}\right) \otimes \mathbb{Q} \leq 1+\operatorname{dim} H_{1}(Y, \mathbb{Q})-2=1 .
$$

Let $(\wedge \alpha, 0)$ be the minimal model for $S^{1}$. We define a DGA map $f: A \rightarrow \wedge \alpha$ by $f\left(t_{i}\right)=f(u)=f(v)=0$ and $f(w)=\alpha$. Moreover define the algebra map $\theta: A \rightarrow \wedge \alpha \otimes A$ by $\theta(w)=1 \otimes w+\alpha \otimes 1$ and $\theta(\gamma)=1 \otimes \gamma$ for $\gamma=t_{1}, t_{2}, u$ and $v$. It is readily seen that $\theta$ is a DGA map. Then the geometrical realizations $|f|: S_{\mathbb{Q}}^{1} \rightarrow Y_{\mathbb{Q}}$ and $|\theta|: S_{\mathbb{Q}}^{1} \times Y_{\mathbb{Q}} \rightarrow Y_{\mathbb{Q}}$ fit into the commutative diagram

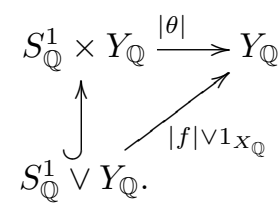

This implies that $|f| \circ e$, the composition of the localization $e: S^{1} \rightarrow S_{\mathbb{Q}}^{1}$ and $|f|$, is a nontrivial Gottlieb element in $\pi_{1}\left(Y_{\mathbb{Q}}\right)$. We conclude that $G_{1}\left(Y_{\mathbb{Q}}\right)=\mathbb{Q}$.

Acknowledgements. The second author thanks Toshihiro Yamaguchi for several helpful conversations during the early stages of this work. The authors thank Gregory Lupton and Samuel Bruce Smith for sending them the preprint [27] and for useful comments on the rational Gottlieb groups. They are grateful to Juno Mukai and Marek Golasiński for comments on the first Gottlieb groups without which Theorem 1.9 could not have been obtained. They also thank the referee for many valuable suggestions to revise a previous version of this paper.

\section{REFERENCES}

[1] Andrews, P. and Arkowitz, M.: Sullivan's minimal models and higher order Whitehead products, Can. J. Math., XXX(1978), 961-982.

[2] Bousfield, A. K. and Gugenheim, V. K. A. M.: On PL de Rham theory and rational homotopy type, Memoirs of AMS 179(1976)

[3] Bousfield, A. K., Peterson, C. and Smith, L: The rational homology of function spaces, Arch. Math. 52 (1989), 275-283

[4] Brown Jr, E. H. and Szczarba, R. H.: Real and Rational Homotopy Theory, in: I.M.James (Ed.), Handbook of Algebraic Topology, Elsevier, Amsterdam, 1995, pp. 867-915.

[5] Brown Jr, E. H. and Szczarba, R. H.: On the rational homotopy type of function spaces, Trans. Amer. Math. Soc. 349(1997), 4931-4951.

[6] Curtis, E. B.: Simplicial Homotopy Theory, Advances in Math. 6(1971), 107-209.

[7] Federer, H.: A study of function spaces by spectral sequences, Trans. Amer. Math. Soc. 82(1956), 340-361.

[8] Félix, Y. and Halperin, S.:Rational L.-S. category and its applications, Trans. Amer. Math. Soc. 273(1982), 1-37.

[9] Félix, Y., Halperin, S. and Thomas, J. -C.: Rational Homotopy Theory, Graduate Texts in Mathematics 205, Springer-Verlag.

[10] Félix, Y. and Lupton, G.: Evaluation maps in rational homotopy, preprint (2005). 
[11] Ghorbal, S.: Monomorphismes et épimorphismes homotopiques, Ph. D. Thesis, Louvain-LaNeuve, 1996.

[12] Gottlieb, D. H.: A certain subgroup of the fundamental group, Amer. J. Math. 87(1965), 840-856.

[13] Gottlieb, D. H.: On fibre spaces and the evaluation map, Ann. of Math. (2)87(1968), 42-55.

[14] Gottlieb, D. H.: Evaluation subgroups of homotopy groups, Amer. J. Math. 91(1969), 729756.

[15] Haefliger, A.:Rational homotopy of space of sections of a nilpotent bundle, Trans. Amer. Math. Soc. 273(1982), 609-620.

[16] Halperin, S.: Lectures on minimal models, Publ. Internes de L'U.E.R. de Math. Pures de l'Université de Lille I, no. 111, 1977.

[17] Halperin, S.: Rational fibrations, minimal models, and fibring of homogeneous spaces, Trans. Amer. Math. Soc. 244(1978), 199-224.

[18] Haslam, H. B.: $G$-spaces mod $F$ and $H$-spaces mod $F$, Duke Math. J. 38(1971), 671-679.

[19] Hilton, P., Mislin, G. and Roitberg, J.: Localization of nilpotent groups and spaces, North Holland Mathematics Studies 15, North Holland, New York, 1975.

[20] Kuribayashi, K.: Mod $p$ equivariant cohomology of homogeneous spaces, J. Pure and Appl. Algebra 147(2000), 95-105.

[21] Kuribayashi, K.: A rational model for the evaluation map, Georgian Mathematical Journal, 13(2006), 127-141.

[22] Lang, G. E.: Localizations and evaluation subgroups, Proc. Amer. Math. Soc. 50(1975), 489-494.

[23] Lee, K. Y. and Woo, M. H.: The $G$-sequence and the $\omega$-homology of a CW-pair, Topology Appl. 52(1993), 221-236.

[24] Lee, K. Y. and Woo, M. H.: Generalized evaluation subgroups of product spaces relative to a factor, Proc. Amer. Math. Soc. 124(1996), 2255-2260.

[25] Lupton, G. and Smith, S. B.: Rationalized evaluation subgroups of a map and the rational $G$-sequence, preprint arXiv:math. AT/0309432(2003), to appear in J. Pure and Appl. Algebra.

[26] Lupton, G and Smith, S. B.: Rationalized evaluation subgroups of a map II: Quillen models and adjoint maps, preprint arXiv:math.AT/0401178(2004), to appear in J. Pure and Appl. Algebra.

[27] Lupton, G and Smith, S.B.: The evaluation subgroup of a fibre inclusion, preprint (2006).

[28] Munkholm, H. J.: The Eilenberg-Moore spectral sequence and strongly homotopy multiplicative maps, J. Pure Appl. Algebra 5(1974), 1-50.

[29] Oda, N.: Pairings and coparings in the category of topological spaces, Publ. Res. Inst. Math. Sci. Kyoto Univ. 28(1992), 83-97.

[30] Oprea, J.:Gottlieb groups, group actions, fix points and rational Homotopy, Lecture Notes Series, 29. Seoul National University, Research Institute of Mathematics, Global Analysis Research Center, Seoul, 1995.

[31] Pan, J. Z. and Woo, M. H.: Exactness of $G$-sequence and monomorphisms, Topology Appl., 109(2001), 315-320

[32] Porter, G.: Higher order Whitehead product, Topology 3 (1965), 123-136.

[33] Siegel, J.: $G$-spaces, $H$-spaces and $W$-spaces, Pacific J. Math., 31(1969), 209-214.

[34] Smith, L.: Homological algebra and the Eilenberg-Moore spectral sequence, Trans. Amer. Math. Soc. 129(1967), 58-93

[35] Smith, S. B.: Rational evaluation subgroups, Math. Z. 221(1996), 387-400.

[36] Smith, S. B.: private communication with the second author (2005)

[37] Stasheff, J.: A classification theorem for fibre spaces, Topology 2(1963), 239-246.

[38] Woo, M. H. and Kim, J. -R.: Certain subgroups of homotopy groups, J. Korean Math. Soc. 21(1984), 109-120.

[39] Woo, M. H. and Kim, J. -R.: Localizations and generalized Gottlieb subgroups, J. Korean Math. Soc. 23(1986), 151-157.

[40] Woo, M. H. and Lee, K. Y.: Homology and generalized evaluation subgroups of homotopy groups, J. Korean Math. Soc. 25(1988), 333-342.

[41] Woo, M. H. and Lee, K. Y.: On the relative evaluation subgroups of a CW-pair, J. Korean Math. Soc. 25(1988), 149-160.

[42] Woo, M. H. and Lee, K. Y.: Exact $G$-sequences and relative $G$-pairs, J. Korean Math. Soc. 27(1990), 177-184. 